\title{
Spectral rigidity and discreteness of 2233-groups
}

\author{
By PETER BUSER, NICOLE FLACH AND KLAUS-DIETER SEMMLER \\ Section de Mathématiques, École Polytechnique Fédérale de Lausanne, \\ Station 8, CH-1015 Lausanne, Switzerland. \\ e-mail: peter.buser@epfl.ch, nicole.flach@bluewin.ch \\ klaus-dieter.semmler@epfl.ch
}

\section{with an Appendix}

\section{By COLIN MACLACHLAN}

Department of Mathematical Sciences, University of Aberdeen, Aberdeen AB24 3UE, Scotland. e-mail: C.Maclachlan@maths.abdn.ac.uk

\section{AND GERHARD ROSENBERGER}

Fachbereich Mathematik, Lehrstuhl LSVI, Universität Dortmund, Vogelpothsweg 87, D-44227 Dortmund, Germany. e-mail: Gerhard.Rosenberger@math.uni-dortmund.de

(Received 10 January 2006; revised 20 February 2007)

Dedicated to the memory of Robert Brooks

\begin{abstract}
In this paper we describe methods for dealing with the trace spectrum of a subgroup of $\operatorname{PSL}(2, \mathbb{R})$ generated by four elliptic elements $\alpha, \beta, \gamma, \delta$ of respective orders $2,2,3,3$, satisfying $\alpha \beta \gamma \delta=1$. We give a parametrization and a fundamental domain in the parameter space of such groups. Furthermore we construct an algorithm that decides whether or not a given group is discrete and which moves the discrete groups into the fundamental domain. Our main result is that any two discrete such groups are isospectral if and only if they are conjugate in GL $(2, \mathbb{R})$.

In the Appendix we consider pairs of subgroups of $\operatorname{PSL}(2, \mathbb{R})$ that arise from nonconjugate maximal orders in a quaternion algebra over a number field. We show that for the isospectrality of such pairs there is a peculiar exception in the case where the groups contain elements of both orders 2 and 3 .
\end{abstract}

\section{Introduction}

In the last 25 years, many examples of pairs of isospectral non-isometric Riemann surfaces have been found, beginning with Vignéras $[\mathbf{3 5 , 3 6}]$ in 1980 and then later by various authors as for instance in [3-6, 33, 34]. In particular, Brooks and Tse [5] have shown that such 
examples exist for any genus $g \geqslant 4$. For $g=2$ and 3 , the existence of such examples is still an open problem.

All constructions use essentially combinatorial methods, and it has been conjectured that for Riemann surfaces or, more generally, quotients of the hyperbolic plane by Fuchsian groups, combinatorics is the only source of isospectrality, and that isospectrality does not occur if there is not enough "room" for combinatorics. Some progress towards showing that sufficiently small topological types are spectrally rigid has been made in $[\mathbf{8}, \mathbf{1 2}, \mathbf{1 8}]$. All known cases, so far, come from 2-generator groups.

In 1994, Maclachlan and Rosenberger [26] described two examples of arithmetic groups of signature $(0 ; 2,2,3,3 ; 0)$ which they claimed to be isospectral. While studying the geometric properties of these examples we found that the trace spectra did not coincide. This led us to the question whether any pair of non-conjugate isospectral examples of this signature exists. We will show that in fact, no such pair exists, and so we have here for the first time, it seems, a full moduli space of spectrally rigid 3-generator groups (Theorems $1 \cdot 3$ and 5.1).

The proof is rather elaborate and made it necessary to describe the geometry of the groups in considerable detail. The paper splits therefore into two main parts. In the first four sections we parameterize all groups of the given type using as parameter space the null set of a certain polynomial. Then we describe an explicit fundamental domain for the Teichmüller modular group in these parameters. That is, we give a complete set of representatives of the conjugacy classes of our groups. This is of interest of its own and is in general quite a complicated task (see e.g. Griffiths [16, 17], Semmler [30], Maskit [28]). A picture of this domain is shown in Figure 2.

In the second main part, Sections 5-7, we show that distinct representatives in the fundamental domain are non-isospectral. It will be shown in Section 5 that out of a properly chosen finite set of traces one can determine the parameters of the group in a purely algebraic way dealing with trace identities and inequalities, but without making any use of the geometry of the underlying groups. In Sections 6 and 7, however, geometry will be needed to make sure that our choice of traces covers the necessary initial part of the spectrum.

Sections 8 and the Appendix give a short account of the examples in [26] which gave rise to this paper.

Let us now introduce the type of groups to be studied.

Definition 1.1. A subgroup $\Gamma$ of $\operatorname{PSL}(2, \mathbb{R})$ is called a 2233-Möbius group if it is generated by four elliptic Möbius transformations $\alpha, \beta, \gamma, \delta$ satisfying

$$
\alpha^{2}=\beta^{2}=\gamma^{3}=\delta^{3}=\alpha \beta \gamma \delta=\mathbf{1}
$$

It is called marked if the list of these generators is explicitly mentioned.

Note that such a group is not necessarily discrete and there may be further independent relations that hold in $\operatorname{PSL}(2, \mathbb{R})$. In particular we do not exclude the case $\alpha=\beta$, so that e.g. the triangle groups $\Gamma(2,3, n)$ are (degenerated) 2233-Möbius groups.

The concept thus set includes the type of groups discussed in Lehner [24], where in addition, $(1 \cdot 1)$ is required to be a faithful representation of an abstractly presented group, as well as the type considered in Singerman [32], where signature $(0 ; 2,2,3,3 ; 0)$ is required, i.e. the group has to be discrete and the quotient of the hyperbolic plane divided by the action of the group must be a closed surface of genus 0 with two cone points of order 2 and two cone points of order 3 . 
We give a representation of the Teichmüller spaces of marked 2233-Möbius groups as null sets of polynomials and an explicit representation of the corresponding modular group in Section 4. Using an algorithm of Nielsen type we then get an explicit description of the moduli space of the discrete 2233-Möbius groups. These fall into three families (Corollary 4.12): the 2-parameter family of Fuchsian groups of signature $(0 ; 2,2,3,3 ; 0)$, the $\Gamma(2,3, n)$ triangle groups, and one elementary group of order 6.

The spectra studied in this paper are the trace spectra. Any $\gamma \in \operatorname{PSL}(2, \mathbb{R})$ is represented by a matrix $X \in \operatorname{SL}(2, \mathbb{R})$ which is determined by $\gamma$ up to multiplication by -1 . We define the trace of $\gamma$ as

$$
\operatorname{tr}(\gamma)=\frac{1}{2}|\operatorname{trace} X|
$$

The factor $1 / 2$ is for practical reasons. Any $\gamma$ acts as an isometry on the Poincaré upper half plane $\mathbb{H}=\{x+i y \in \mathbb{C} \mid y>0\}$ with respect to the hyperbolic metric, $\operatorname{and} \operatorname{tr}(\gamma)$ is the cosine of half the rotational angle if $\gamma$ is elliptic, respectively the hyperbolic cosine of half the displacement length if $\gamma$ is hyperbolic.

Definition $1 \cdot 2$. For any finitely generated discrete subgroup $\Gamma \subset \operatorname{PSL}(2, \mathbb{R})$ we consider the sets

$$
C(\Gamma)=\{[\gamma] \mid \gamma \in \Gamma\} \quad \text { and } \quad C^{\prime}(\Gamma)=\left\{[\gamma]^{\prime} \mid \gamma \in \Gamma\right\},
$$

where $[\gamma]$ is the conjugacy class of $\gamma$ in $\Gamma$, and $[\gamma]^{\prime}=[\gamma] \cup\left[\gamma^{-1}\right]$ is called the extended conjugacy class. The trace spectra of $\Gamma$ are the following sequences listed in increasing order and with multiplicities

$$
\operatorname{TS}(\Gamma)=\{\operatorname{tr}(\gamma) \mid[\gamma] \in C(\Gamma)\} \quad \text { and } \quad \operatorname{TS}^{\prime}(\Gamma)=\left\{\operatorname{tr}(\gamma) \mid[\gamma]^{\prime} \in C^{\prime}(\Gamma)\right\} .
$$

We will call $\mathrm{TS}(\Gamma)$ the algebraic trace spectrum and $\mathrm{TS}^{\prime}(\Gamma)$ the geometric trace spectrum.

Observe that $\operatorname{TS}(\Gamma)$ contains each member of $\operatorname{TS}^{\prime}(\Gamma)$ twice except for the ones corresponding to elements of order two or to elements of the form $\gamma=\sigma \tau$ with $\sigma^{2}=\tau^{2}=\mathbf{1}$. While $\operatorname{TS}^{\prime}(\Gamma)$ is more natural from a geometric point of view, $\operatorname{TS}(\Gamma)$ is used for example in the Selberg trace formula (see Hejhal [19, chapter 3, theorem 5·1]).

Our main result is the following, where we note that $\operatorname{PGL}(2, \mathbb{R})$ may be identified with the isometry group of $\mathbb{H}$ (in which we include the orientation reversing isometries).

THEOREM 1.3. Let $\Gamma_{1}, \Gamma_{2}$ be discrete 2233-Möbius groups. If either $\operatorname{TS}\left(\Gamma_{1}\right)=\operatorname{TS}\left(\Gamma_{2}\right)$ or $\operatorname{TS}^{\prime}\left(\Gamma_{1}\right)=\operatorname{TS}^{\prime}\left(\Gamma_{2}\right)$, then $\Gamma_{1}$ and $\Gamma_{2}$ are conjugate in $\operatorname{PGL}(2, \mathbb{R})$.

\section{Möbius groups and matrix groups}

For computational matters it is useful to work with matrices, and so all groups will be lifted from $\operatorname{PSL}(2, \mathbb{R})$ to $\operatorname{SL}(2, \mathbb{R})$. We first collect some general facts about traces of the corresponding matrices.

Definition $2 \cdot 1$. For any $2 \times 2$ matrix $X \in \mathrm{M}(2, \mathbb{R})$ we write

$$
\operatorname{tr}: \mathrm{M}(2, \mathbb{R}) \longrightarrow \mathbb{R}, \quad \operatorname{tr}(X):=\frac{1}{2} \operatorname{trace}(X) .
$$

Note that these traces have signs. We point out that although the signs are of no significance for the corresponding Möbius transformations, there are situations (e.g. Observation 3.3) where they do carry geometric information. 
In the following lemma we collect trace identities that go back to Fricke-Klein [15]. A more recent reference with applications to spectral questions is Horowitz [22, p. 637]. Relations of this type are used extensively in Helling [20, 21]. Of course, the lemma may be checked by direct computation.

LEMmA 2.2. If $A, B, C, X, Y \in \mathrm{SL}(2, \mathbb{R})$ and $\operatorname{tr}(A)=\operatorname{tr}(B)=0$, then

(i) $\operatorname{tr}\left(X Y^{-1}\right)=2 \operatorname{tr}(X) \operatorname{tr}(Y)-\operatorname{tr}(X Y)$,

(ii) $\operatorname{tr}(A B C)+\operatorname{tr}(A C B)=2 \operatorname{tr}(C) \operatorname{tr}(A B)$,

(iii) $\operatorname{tr}(A B C) \operatorname{tr}(A C B)=\operatorname{tr}^{2}(C)-1+\operatorname{tr}^{2}(A B)+\operatorname{tr}^{2}(B C)+\operatorname{tr}^{2}(C A)$

$$
+2 \operatorname{tr}(A B) \operatorname{tr}(B C) \operatorname{tr}(C A) \text {. }
$$

We will lift the generators of 2233-Möbius groups to SL $(2, \mathbb{R})$ with the help of the following observation (see e.g. Milnor [29]).

FACT 2.3. For $X \in \operatorname{SL}(2, \mathbb{R})$ and $n>0$ minimal such that $X^{n} \in\{-\mathbf{1}, \mathbf{1}\}$ we have

$$
\operatorname{tr}(X) \geqslant 0 \quad \Longrightarrow \quad X^{n}=-\mathbf{1} .
$$

Definition 2.4. A subgroup $G$ of $\operatorname{SL}(2, \mathbb{R})$ is called a 2233-matrix group if it is generated by four matrices $A, B, C, D \in \mathrm{SL}(2, \mathbb{R})$ of non-negative traces satisfying

$$
\begin{array}{r}
A^{2}=-\mathbf{1}, \quad B^{2}=-\mathbf{1}, \quad C^{3}=-\mathbf{1}, \quad D^{3}=-\mathbf{1}, \quad A B C D=\boldsymbol{\epsilon} \mathbf{1}, \\
\text { where } \epsilon \in\{-1,1\} .
\end{array}
$$

The group is called marked if it is given together with the ordered list of these generators. Two marked groups are marking equivalent if they differ by a conjugation in $\operatorname{GL}(2, \mathbb{R})$ that conjugates the ordered lists of the generators.

Notation 2.5. In this paper, the symbol $\epsilon$ will always refer to the sign appearing in the above definition.

For any 2233-Möbius group with a given choice of generators $\alpha, \beta, \gamma, \delta$, we find matrices $A, B, C, D \in \operatorname{SL}(2, \mathbb{R})$ that project to $\alpha, \beta, \gamma, \delta$ under the natural projection $\operatorname{SL}(2, \mathbb{R}) \rightarrow$ $\operatorname{PSL}(2, \mathbb{R})$, and thus generate a 2233-matrix group by Fact 2.3. The matrices with trace 0 in this sequence are only determined up to a multiplication by -1 . To make them unique, we require an additional condition,

$$
\operatorname{tr}(C)>0, \quad \operatorname{tr}(D)>0, \quad \operatorname{tr}(A C)<0, \quad \operatorname{tr}(B C)<0 .
$$

If this is satisfied we shall say that the ordered sequence $A, B, C, D$ is a standard marking of the matrix group. The next observation allows us to define parameters for Möbius groups using these matrices.

OBSERVATION 2.6. Any 2233-Möbius group lifts to a 2233-matrix group with standard marking. If $\Gamma_{1}, \Gamma_{2}$ with generators $\alpha_{i}, \beta_{i}, \gamma_{i}, \delta_{i}$, are conjugate in $\operatorname{PGL}(2, \mathbb{R})$ by a conjugation that sends $\alpha_{1}, \beta_{1}, \gamma_{1}, \delta_{1}$ to $\alpha_{2}, \beta_{2}, \gamma_{2}, \delta_{2}$, and if $A_{i}, B_{i}, C_{i}, D_{i}$ are the lifts of the generators in $\in \mathrm{SL}(2, \mathbb{R})$ satisfying the sign convention $(2 \cdot 2)$, then there exists a conjugation in $\mathrm{GL}(2, \mathbb{R})$ sending $A_{1}, B_{1}, C_{1}, D_{1}$ to $A_{2}, B_{2}, C_{2}, D_{2}$.

Proof. The appearance of $\operatorname{GL}(2, \mathbb{R})$ rather than $\mathrm{SL}(2, \mathbb{R})$ comes from the fact that conjugation of Möbius groups takes place in the full isometry group of $\mathbb{H}$, where we also have orientation reversing elements. The observation follows from the identification of Isom( $(\mathbb{H})$ with $\operatorname{PGL}(2, \mathbb{R})$ (e.g. [31]). 
Remark 2.7. A comment on our use of the word "lift" may be necessary. If $\Gamma$ is a 2233 Möbius group and $G$ its lift as described above, then the natural projection from $G$ to $\Gamma$ is 2-to-1. Furthermore, the generators of $G$ are of order 4 and 6 , so the two groups are not isomorphic. Hence "lift" is not synonymous with "embedding". It is shown in [31, sections 3.20-3.22], that the above construction leads to an embedding of a group of Möbius transformations in $\operatorname{SL}(2, \mathbb{R})$ if and only if this group contains no elements of order two.

The fact that our lifts are 2-to-1 rather than 1-to-1 has no influence in what follows.

Möbius groups such as $\Gamma_{1}, \Gamma_{2}$ in Observation 2.6 are called marking equivalent. Thus, the marking equivalence classes of the 2233-Möbius groups may be identified with the marking equivalence classes of the 2233-matrix groups with standard marking.

PROPOSITION 2.8. The generators of a marked 2233-matrix group with sign convention (2.2) satisfy:

$$
\begin{gathered}
\operatorname{tr}(A)=\operatorname{tr}(B)=0, \quad \operatorname{tr}(C)=\operatorname{tr}(D)=\frac{1}{2} \\
\operatorname{tr}(A B) \leqslant-1, \quad \operatorname{tr}(B C) \leqslant-\frac{1}{2} \sqrt{3}, \quad \operatorname{tr}(C A) \leqslant-\frac{1}{2} \sqrt{3} \\
-\epsilon \frac{1}{2} \operatorname{tr}(A B)+2 \operatorname{tr}(B C) \operatorname{tr}(C A) \operatorname{tr}(A B) \\
+\operatorname{tr}^{2}(B C)+\operatorname{tr}^{2}(C A)+\operatorname{tr}^{2}(A B)-\frac{1}{2}=0 \\
-2 \operatorname{tr}(B C) \operatorname{tr}(C A) \operatorname{tr}(A B)-\operatorname{tr}^{2}(B C)-\operatorname{tr}^{2}(C A) \geqslant \frac{3}{4}\left(\operatorname{tr}^{2}(A B)-1\right) \geqslant 0 .
\end{gathered}
$$

Proof. (1) is clear. Conjugating the triple $A, B, C$ in $\mathrm{GL}(2, \mathbb{R})$ as e.g. in the next section, we easily check that $|\operatorname{tr}(A B)| \geqslant 1$ and $|\operatorname{tr}(B C)|,|\operatorname{tr}(C A)| \geqslant(1 / 2) \sqrt{3}$. The second and the third inequality in (2) thus follow from the sign convention $(2 \cdot 2)$.

(3) is Lemma $2 \cdot 2$ (ii), (iii) with $A B C=\epsilon D^{-1}$, and (4) follows from (3) writing

$$
\operatorname{tr}^{2}(A B)-\epsilon \frac{1}{2} \operatorname{tr}(A B)-\frac{1}{2}=\frac{3}{4}\left(\operatorname{tr}^{2}(A B)-1\right)+\frac{1}{4}(\operatorname{tr}(A B)-\epsilon)^{2} .
$$

Finally, (4) yields $\operatorname{tr}(A B)<0$, hence the first inequality in (2).

Definition 2.9. We define three real parameters to describe a 2233-matrix group with standard marking $\{A, B, C, D\}$,

$$
x=-\operatorname{tr}(A B), \quad y=-\operatorname{tr}(B C), \quad z=-\operatorname{tr}(C A),
$$

and, by Proposition $2 \cdot 8$, use them in the parameter space $\{(x, y, z) \mid x \geqslant 1, y, z \geqslant \sqrt{3} / 2\}$. By Observation 2.6, these parameters describe also the marked 2233-Möbius groups.

We will show in Section 3 that up to conjugation in $\operatorname{GL}(2, \mathbb{R})$, there is at most one 2233matrix group with standard marking, for any triple $(x, y, z)$ in the above parameter space (Proposition 3·2).

\section{Explicit matrices}

In this section we calculate explicitly matrices for 2233-matrix groups. This is used for existence proofs. Otherwise the section is independent of the rest of the paper because all the information needed is coded in the variables $x, y, z, \boldsymbol{\epsilon}$. 
PROPOSITION 3.1. For three positive real numbers

$$
(x, y, z) \text { with } x>1 \text { and } 2 x y z-z^{2}-y^{2} \geqslant \frac{3}{4}\left(x^{2}-1\right),
$$

respectively $x=1$ and $y=z \geqslant \sqrt{3} / 2$, there exist three matrices $A, B, C \in \operatorname{SL}(2, \mathbb{R})$ having the properties

$$
\begin{aligned}
\operatorname{tr}(A) & =0 & \operatorname{tr}(B) & =0 & \operatorname{tr}(C) & =\frac{1}{2} \\
\operatorname{tr}(A B) & =-x & \operatorname{tr}(B C) & =-y & \operatorname{tr}(C A) & =-z .
\end{aligned}
$$

The triple $A, B, C$ is unique up to conjugation in $\mathrm{GL}(2, \mathbb{R})$ or passing simultaneously to the inverses.

Proof. Uniqueness: $A, B, C$ are elliptics. We conjugate the triple such that the fixed points of $A$ and $B$ in the upper half plane become $i$ and $\lambda i$ with $\lambda \geqslant 1$ respectively. Then we conjugate again, using reflection along the imaginary axis so that the fixed point of $C$ becomes $r+i s$ with $s>0, r \leqslant 0$. This reflection is obtained by conjugation with $\left(\begin{array}{ll}1 & 0 \\ 0 & -1\end{array}\right)$. In the special case where $x=1$, and thus $A=B$, we further apply a rotation around $i$ so that the fixed point of $C$ in $\mathbb{H}$ becomes $i s$ with $s \geqslant 1$. The resulting matrices are again called $A, B, C$. They are

$$
\begin{array}{ll}
A=\left(\begin{array}{cc}
0 & \sigma \\
-\sigma & 0
\end{array}\right) & B=\left(\begin{array}{cc}
0 & \lambda \sigma \\
-\frac{\sigma}{\lambda} & 0
\end{array}\right) \\
C=\frac{1}{2 s}\left(\begin{array}{cc}
s-r \varrho \sigma & \left(r^{2}+s^{2}\right) \varrho \sigma \\
-\varrho \sigma & s+r \varrho \sigma
\end{array}\right), &
\end{array}
$$

where $\varrho^{2}=3$ and $\sigma^{2}=1$. Note that changing the sign of $\sigma$ we pass simultaneously to the inverses. The negative traces of the products become

$$
x=\frac{1}{2}\left(\frac{1}{\lambda}+\lambda\right), \quad y=\frac{\varrho\left(r^{2}+s^{2}+\lambda^{2}\right)}{4 \lambda s}, \quad z=\frac{\varrho\left(r^{2}+s^{2}+1\right)}{4 s} .
$$

Now, $\lambda \geqslant 1$ is determined by $x$. The sign of $s$ yields $\varrho=\sqrt{3}$. In the case $x=1$ we have $\lambda=1, y=z, r=0$, and $s$ is the larger solution to $(1 / s+s)=4 y / \sqrt{3}$. In the case $x>1$ the quantities $y / z$ and $\lambda$ determine $r^{2}+s^{2}$, and then $z$ determines $s>0$ and therefore also $r \leqslant 0$. Hence the equations (3.2) can be solved for $\lambda \geqslant 1, s>0, r \leqslant 0$ uniquely:

$$
\lambda=x+\sqrt{x^{2}-1}, \quad r^{2}+s^{2}=\frac{\lambda-\frac{y}{z}}{\frac{y}{z}-\frac{1}{\lambda}}, \quad s=\frac{\sqrt{3}\left(r^{2}+s^{2}+1\right)}{4 z} .
$$

Existence: If $x=1$, then by hypothesis $y \geqslant \sqrt{3} / 2$, and the equation $(1 / s+s)=4 y / \sqrt{3}$ has a solution $s \geqslant 1$. If $x>1$, then (3.3) has a solution for $r$ and $s$ when $u=(\lambda-y / z) /(y / z-1 / \lambda) \geqslant s^{2}=3(u+1)^{2} /\left(16 z^{2}\right)$, and this inequality is equivalent to $2 x y z-z^{2}-y^{2} \geqslant 3\left(x^{2}-1\right) / 4$.

Observe that with the above matrices, $D:=\boldsymbol{\epsilon}(A B C)^{-1}$ turns out to be

$$
D=\frac{-\epsilon}{2 s}\left(\begin{array}{cc}
(s+r \sigma \sqrt{3}) \lambda & \frac{-\left(r^{2}+s^{2}\right) \sigma \sqrt{3}}{\lambda} \\
\lambda \sigma \sqrt{3} & \frac{(s-r \sigma \sqrt{3})}{\lambda}
\end{array}\right),
$$

and $\operatorname{tr}(D)$ is a solution to the polynomial equation

$$
\operatorname{tr}^{2}(D)+\epsilon x \operatorname{tr}(D)+x^{2}+y^{2}+z^{2}-2 x y z-\frac{3}{4}=0
$$



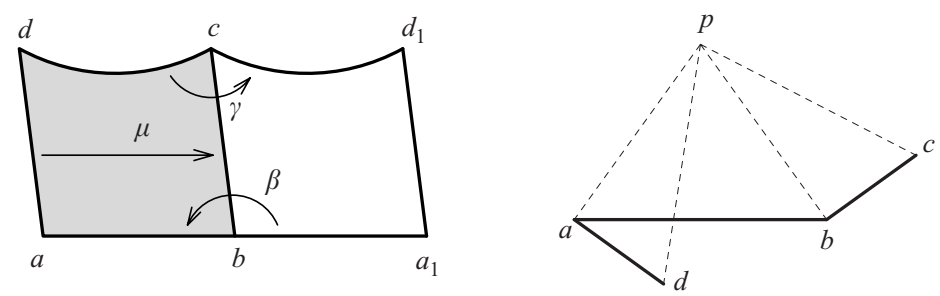

Fig. 1.

Proposition 3.2. Let $A, B, C$ be as in Proposition $3 \cdot 1$ and assume that for $D:=$ $\boldsymbol{\epsilon}(A B C)^{-1}$ we have $\operatorname{tr}(D)=1 / 2$.

If $x=1$, then $\epsilon=-1, A=B, C^{-1}=D$, and the couple $B, C$ is uniquely determined by $y$ up to conjugation in $\operatorname{GL}(2, \mathbb{R})$.

If $x>1$, then the quadruple $A, B, C, D$ is uniquely determined by $(x, y, z, \boldsymbol{\epsilon})$ up to conjugation in $\mathrm{GL}(2, \mathbb{R})$.

Proof. Conjugate $A, B, C$ as in the proof of Proposition 3.1. The only parameter then left free is the sign of $\sigma$.

For $x=1$ we have $\lambda=1, r=0$, and $\operatorname{tr}(D)=-\epsilon / 2$. Hence, $\epsilon=-1$, and changing the sign of $\sigma$ can be obtained by conjugating $A, B, C$ with $\left(\begin{array}{cc}1 & 0 \\ 0 & -1\end{array}\right)$.

For $x>1$, only $\sigma=+1$ is possible because otherwise we get $|\operatorname{tr} D| \geqslant(1 / \lambda+\lambda) / 4$.

We add some remarks about the fixed points of the above generators.

If in (3.4) we have $\operatorname{tr}(D)=1 / 2$, then $s=-r \sqrt{3}(\lambda-\epsilon) /(\lambda+\epsilon)$, and the fixed point of $D$ in $\mathbb{H}$ becomes $(\epsilon r+i s) / \lambda$. Hence, the fixed points of the generators form the following polygon:

$$
a=i, \quad b=\lambda i, \quad c=r+i s, \quad d=\frac{1}{\lambda}(\epsilon r+i s) .
$$

Translating this to Möbius groups we get the following.

OBSERVATION 3.3. Let $\alpha, \beta, \gamma, \delta$ with $\alpha^{2}=\beta^{2}=\gamma^{3}=\delta^{3}=\alpha \beta \gamma \delta=\mathbf{1}$ be the generators of a 2233-Möbius group, take the lifts $A, B, C, D$ in $\operatorname{SL}(2, \mathbb{R})$ with sign convention (2.2) and set $\epsilon$ such that $A B C D=\epsilon \mathbf{1}$. Then the polygon abcd formed by the fixed points of $\alpha, \beta, \gamma, \delta$ respectively, is convex if $\epsilon=1$ and crossed if $\epsilon=-1$.

"Crossed" is short hand for the three remaining cases: self-crossing, non-convex and degenerate. It is interesting to note that this way of reading off the convexity is inaccessible in the Möbius group itself.

Figure 1 shows the two cases schematically. Denoting by $\mu$ the hyperbolic isometry with axis through $a, b$ which shifts $a$ to $b$ (and thus $\mu^{2}=\beta \alpha$ ), and by $\eta$ the symmetry with respect to this axis, we have

$$
\mu(d)=c, \text { if } \epsilon=1, \quad \mu \eta(d)=c, \text { if } \epsilon=-1 .
$$

In the first case, the filled quadrilateral abcd and its image under $\mu$ together form a polygon domain $\mathcal{P}=a b a_{1} d_{1} c d$ with $a_{1}=\beta \alpha(a), d_{1}=\beta \alpha(d)$. The group $\Gamma$ generated by $\alpha, \beta, \gamma, \delta$ or likewise $\mu, \beta, \gamma$ has the following geometric property.

Proposition 3.4. P is a fundamental domain for the action of $\Gamma$ on $\mathbb{H}$, and $\mathbb{H} / \Gamma$ is an

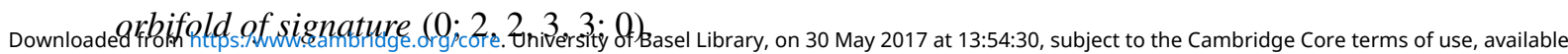


Proof. This follows from Poincaré's theorem $[\mathbf{1}, \mathbf{1 1}, \mathbf{2 7}]$ : Since $\alpha \beta \gamma \delta=\mathbf{1}$, we have $d_{1}=$ $\beta \alpha(d)=\beta \alpha \delta^{-1}(d)=\gamma(d)$, and so the generators $\beta, \gamma, \mu=\beta \alpha$ of $\Gamma$ yield the side pairing

$$
\beta\left(b a_{1}\right)=b a, \quad \mu(a d)=a_{1} d_{1}, \quad \gamma(c d)=c d_{1} .
$$

Furthermore, since $\mu(a b c d)=b a_{1} d_{1} c$, the sum of the interior angles of $\mathcal{P}$ at $a$ and $a_{1}$ equals the angle at $b$ which is $\pi$, and the sum of the angles at $d$ and $d_{1}$ equals the angle at $c$ which is $2 \pi / 3$.

The right hand side of Figure 1 depicts a case for $\epsilon=-1$, where $B C$ is elliptic with some fixed point $p$. Since $B C=-\epsilon A D^{-1}=A D^{-1}, p$ is also the fixed point of $A D^{-1}$. (In the figure, $C$ rotates counter-clockwise, and $D$ clock-wise.) This case will be discussed in Section $4 \cdot 3$ (see the remarks after the proof of Observation 4.10).

\section{Null sets of polynomials as Teichmüller spaces}

We now introduce the Teichmüller spaces of the marked 2233-Möbius groups for $\epsilon=-1$ and 1, following the approach of Helling [20, 21]. In Section 4.2 the modular group is presented by natural polynomial actions. In Section 4.3 we describe an explicit fundamental domain $\mathcal{F}_{\epsilon}$ for the action of the modular group on the discrete locus and define an algorithm which a) determines whether or not a given 2233-group is discrete and b) constructs its representative in $\mathcal{F}_{\epsilon}$ in case it is discrete. Section 4.4 lists properties of $\mathcal{F}_{1}$ needed for the discussion of the trace spectra.

\subsection{Teichmüller spaces}

Definition 4.1. We define the following polynomials and their null sets for $\epsilon= \pm 1$.

$$
\begin{aligned}
P_{\epsilon}(x, y, z) & :=x^{2}+y^{2}+z^{2}-2 x y z+\frac{\epsilon x-1}{2}, \\
\mathcal{T}_{\epsilon} & :=\left\{(x, y, z) \mid x \geqslant 1, y, z \geqslant \sqrt{3} / 2, P_{\epsilon}(x, y, z)=0\right\} .
\end{aligned}
$$

Proposition 4.2. $(x, y, z)$ with $x \geqslant 1, y, z \geqslant \sqrt{3} / 2$ are the parameters of a 2233matrix group as in Definition 2.9 iff they satisfy

$$
P_{\epsilon}(x, y, z)=0 .
$$

In this sense the sets $\mathcal{T}_{\epsilon}$ serve as Teichmüller spaces for the 2233-matrix groups marked by the choice of generators satisfying $(2 \cdot 1)$ and $(2 \cdot 2)$, or, what is the same (see Observation $2 \cdot 6$ ), for the marking equivalence classes of the 2233-Möbius groups. Note that the groups need not be discrete.

Proof. For a 2233-matrix group the above polynomials are the relations 2.8(3). Conversely, given $(x, y, z)$ satisfying $P_{\epsilon}(x, y, z)=0$, construct matrices as in Proposition $3 \cdot 1$ (we have $\left.2 x y z-y^{2}-z^{2}=(3 / 4)\left(x^{2}-1\right)+(1 / 4)(x-\boldsymbol{\epsilon})^{2}\right)$ with $\sigma=1$, to obtain generators $A, B, C$ and put $D=\epsilon(A B C)^{-1}$. Now $x^{2}+y^{2}+z^{2}-2 x y z=-(\epsilon x-1) / 2$, and equation (3.5) becomes

$$
0=\operatorname{tr}^{2}(D)+\epsilon x \operatorname{tr}(D)-\frac{1}{2}(\epsilon x-1)-\frac{3}{4}=\left(\operatorname{tr}(D)-\frac{1}{2}\right)\left(\operatorname{tr}(D)+\epsilon x+\frac{1}{2}\right) .
$$

By (3.4) and using that $\sigma=1$ and $\lambda \geqslant 1$, we have $\epsilon \operatorname{tr}(D) \geqslant-(1 / 2) x$. Hence, $\boldsymbol{\epsilon}(\operatorname{tr}(D)+\boldsymbol{\epsilon} x+$ $1 / 2) \geqslant(1 / 2)(x+\boldsymbol{\epsilon})$, where the right-hand side is positive except for the case $x=1, \boldsymbol{\epsilon}=-1$. It follows that $\operatorname{tr}(D)=1 / 2$, and the construction is complete. 


\subsection{The Teichmüller-modular group in these parameters}

There is an obvious action on the null sets of our quadratic polynomials: If $(x, y, z) \in \mathcal{T}_{\epsilon}$ then

other z: $\quad\left(x, y, z^{\prime}\right) \in \mathcal{T}_{\boldsymbol{\epsilon}}, \quad$ where $\quad z^{\prime}=2 x y-z$,

other y: $\quad\left(x, y^{\prime}, z\right) \in \mathcal{T}_{\boldsymbol{\epsilon}}, \quad$ where $\quad y^{\prime}=2 x z-y$,

other $\mathbf{x}: \quad\left(x^{\prime}, y, z\right) \in \mathcal{T}_{\epsilon}, \quad$ where $\quad x^{\prime}=2 y z-\frac{1}{2} \boldsymbol{\epsilon}-x$,

y-z-symmetry: $\quad(x, z, y) \in \mathcal{T}_{\boldsymbol{\epsilon}}$.

In fact, fixing any two of the variables, $P_{\epsilon}$ becomes a quadratic polynomial in the third, and if that one is a root, its prime is the other root. The only point to check is that the two roots lie on the same side of 1 in case this variable is $x$, and on the same side of $\sqrt{3} / 2$ in case the variable is $y$ or $z$. For this it suffices to observe that

$$
\begin{gathered}
P_{\epsilon}(1, y, z)=(z-y)^{2}+\frac{1}{2}(\boldsymbol{\epsilon}+1) \geqslant 0, \\
P_{\epsilon}\left(x, y, \frac{\sqrt{3}}{2}\right)=\left(y-\frac{\sqrt{3}}{2} x\right)^{2}+\frac{1}{4}(x+\epsilon)^{2} \geqslant 0 .
\end{gathered}
$$

The first line is strictly positive except for the case $\epsilon=-1, y=z$, in which the two roots are $x=1, x^{\prime}=2 y^{2}-1 / 2 \geqslant 1$. The second line is strictly positive except for the case $\boldsymbol{\epsilon}=-1, x=1, y=\sqrt{3} / 2$, in which the two roots are $z=\sqrt{3} / 2=z^{\prime}$.

Definition 4.3. The four transformations other $\mathbf{z}: \mathcal{T}_{\boldsymbol{\epsilon}} \rightarrow \mathcal{T}_{\boldsymbol{\epsilon}},(x, y, z) \mapsto\left(x, y, z^{\prime}\right)$, etc. generate a group of automorphisms of $\mathcal{T}_{\epsilon}$. This group is denoted by $\mathcal{M}_{\epsilon}$.

We show that $\mathcal{M}_{\epsilon}$ corresponds to the change of markings i.e. change of generators satis-

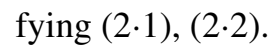

THEOREM 4.4. If $(x, y, z)$ and $\left(x_{1}, y_{1}, z_{1}\right)$ lie on the same orbit w.r.t.the action of $\mathcal{M}_{\epsilon}$ on $\mathcal{T}_{\epsilon}$, then the corresponding 2233-Möbius groups are conjugate in $\operatorname{Isom}(\mathbb{H})$.

Proof. By Observation 2.6, one may work with matrix groups. We will show that for any automorphism $m \in \mathcal{M}_{\epsilon}$ there are words $w_{1}, w_{2}, w_{3}, w_{4}$ over an alphabet of four letters such that if a 2233-matrix group has standard marking $A, B, C, D$ with parameters $(x, y, z)$ (Definition 2.9), then the $w_{i}(A, B, C, D)$ form a standard marking of the same group with parameters $m(x, y, z)$. Of course, this program needs to be carried out only for the generators of $\mathcal{M}_{\epsilon}$.

y-z-symmetry: we put

$$
A_{1}:=-B, \quad B_{1}:=-A, \quad C_{1}:=C^{-1}, \quad D_{1}:=C D^{-1} C^{-1} .
$$

(2.1) is easily checked for the new generators, and furthermore, $\operatorname{tr}\left(C_{1}\right)=\operatorname{tr}\left(D_{1}\right)=1 / 2$. Using 2.2(i), we obtain the following relations, which also imply the two remaining conditions in $(2 \cdot 2)$,

$$
x_{1}=-\operatorname{tr}\left(A_{1} B_{1}\right)=x, \quad y_{1}=-\operatorname{tr}\left(B_{1} C_{1}\right)=z, \quad z_{1}=-\operatorname{tr}\left(C_{1} A_{1}\right)=y .
$$

other $\mathbf{z}$ : here we put

$$
A_{1}:=-\epsilon B, \quad B_{1}:=-\epsilon A, \quad C_{1}:=D^{-1}, \quad D_{1}:=C^{-1} .
$$

(2.1) is checked again for the new generators, and $\operatorname{tr}\left(C_{1}\right)=\operatorname{tr}\left(D_{1}\right)=1 / 2$. Using $2 \cdot 2(\mathrm{i})$ and 
$\epsilon D^{-1}=A B C$, we obtain

$$
\begin{aligned}
x_{1} & =-\operatorname{tr}\left(A_{1} B_{1}\right)=-\operatorname{tr}(B A)=x, \\
y_{1} & =-\operatorname{tr}\left(B_{1} C_{1}\right)=-\operatorname{tr}\left(-A \epsilon D^{-1}\right)=-\operatorname{tr}(B C)=y, \\
z_{1} & =-\operatorname{tr}\left(C_{1} A_{1}\right)=-\operatorname{tr}\left(-B \epsilon D^{-1}\right)=\operatorname{tr}(B A B C), \\
& =2 \operatorname{tr}(B A) \operatorname{tr}(B C)-\operatorname{tr}(A B B C)=2 x y-z .
\end{aligned}
$$

This is the required action, and since we already know it operates in $\mathcal{T}_{\boldsymbol{\epsilon}}$, we see here that $\operatorname{tr}\left(A_{1} C_{1}\right)<0$ and $\operatorname{tr}\left(B_{1} C_{1}\right)<0$.

other $\mathbf{y}$ : this transformation can be obtained by using first $\mathbf{y}-\mathbf{z}$-symmetry followed by other $\mathbf{z}$ and then again $\mathbf{y}$-z-symmetry.

The most interesting transformation is

other $\mathbf{x}$ : here we put

$$
A_{1}:=-A, \quad B_{1}:=-C^{-1} B C, \quad C_{1}:=C^{-1}, \quad D_{1}:=A D^{-1} A^{-1} .
$$

$(2 \cdot 1)$ and $\operatorname{tr}\left(C_{1}\right)=\operatorname{tr}\left(D_{1}\right)=1 / 2$ are checked once again, and using $2 \cdot 2(\mathrm{i})$ and $\epsilon D^{-1}=$ $A B C$, we get

$$
\begin{aligned}
x_{1} & =-\operatorname{tr}\left(A_{1} B_{1}\right)=-\operatorname{tr}\left(A C^{-1} B C\right)=-\left(2 \operatorname{tr}\left(A C^{-1}\right) \operatorname{tr}(B C)-\operatorname{tr}\left(C A^{-1} B C\right)\right), \\
& =2 y z-\left(2 \operatorname{tr}(C) \operatorname{tr}(A B C)-\operatorname{tr}\left(C^{-1} A B C\right)\right)=2 y z-\frac{1}{2} \epsilon-x, \\
y_{1} & =-\operatorname{tr}\left(B_{1} C_{1}\right)=-\operatorname{tr}\left(-C^{-1} B\right)=-\operatorname{tr}(C B)=y, \\
z_{1} & =-\operatorname{tr}\left(C_{1} A_{1}\right)=-\operatorname{tr}\left(-C^{-1} A\right)=-\operatorname{tr}(C A)=z .
\end{aligned}
$$

This is indeed the other $\mathbf{x}$-action. Hence, $\left(x_{1}, y_{1}, z_{1}\right) \in \mathcal{T}_{\boldsymbol{\epsilon}}$ and $\operatorname{tr}\left(A_{1} C_{1}\right)<0, \operatorname{tr}\left(B_{1} C_{1}\right)<0$.

For easy reference we restate the expressions for $x^{\prime}$ and $z^{\prime}$,

$$
x^{\prime}=2 y z-\frac{1}{2} \epsilon-x=-\operatorname{tr}\left(B C A C^{-1}\right), \quad z^{\prime}=2 x y-z=\operatorname{tr}(B A B C) .
$$

\subsection{Fundamental domains}

Definition $4 \cdot 5$. We define the following domains in $\mathcal{T}_{\epsilon}$,

$$
\mathcal{F}_{\boldsymbol{\epsilon}}:=\left\{(x, y, z) \in \mathcal{T}_{\boldsymbol{\epsilon}} \mid y \leqslant z \leqslant x y, \quad x \leqslant y z-\frac{1}{4} \boldsymbol{\epsilon}\right\} .
$$

Rewriting the polynomials $\mathcal{P}_{\epsilon}$ in the form

$$
\begin{aligned}
P_{\boldsymbol{\epsilon}}(x, y, z)= & (z-x y)^{2}+\frac{\boldsymbol{\epsilon} x+1}{2}-\left(x^{2}-1\right)\left(y^{2}-1\right), \quad \boldsymbol{\epsilon}= \pm 1, \\
P_{-1}(x, y, z)= & -\left[(x y-z)(z-y)+\left(\left(y z+\frac{1}{4}\right)-x\right)(x-1)\right. \\
& \left.+\left(y^{2}-\frac{3}{4}\right)(x-1)\right],
\end{aligned}
$$

we get the following properties.

Proposition 4.6. If $(x, y, z) \in \mathcal{T}_{1}$, then

$$
x, y, z>1 \text { and }(x-1)\left(y^{2}-1\right) \geqslant \frac{1}{2} .
$$

If $(x, y, z) \in \mathcal{T}_{-1}$, then $(x+1)\left(1-y^{2}\right) \leqslant 1 / 2$ and

$$
(x, y, z) \in \mathcal{F}_{-1} \Longleftrightarrow x=1 \Longleftrightarrow z=y=x y .
$$


Proof. By (4.3), the conditions $x-1, y, z \geqslant 0$ imply $x, y>1$ for any solution of $P_{1}(x, y, z)=0$. By symmetry (in the original writing of $P_{1}$ ) also $z>1$. The second statement for $\mathcal{T}_{1}$ and the first statement for $\mathcal{T}_{-1}$ follow from (4.3) in the same way.

For the equivalences we begin with $(x, y, z) \in \mathcal{F}_{-1}$. In this case all three terms in the brackets of (4.4) are non-negative, so they vanish, and we proceed with the following logical game: if $x>1$, then $y=\sqrt{3} / 2$ and $x=(\sqrt{3} / 2) z+1 / 4$, and either of the conditions $z=y=\sqrt{3} / 2$ and $z=x y=(\sqrt{3} / 2) x$ implies $x=1$, a contradiction, hence $x=1$.

If $x=1$, then (4.4) yields $z=y=x y$, and by the definition of $\mathcal{F}_{-1}$ this implies $(x, y, z) \in$ $\mathcal{F}_{-1}$.

We will show that $\mathcal{F}_{\epsilon}$ is a fundamental domain for $\mathcal{M}_{\epsilon}$ for the discrete locus i.e. the part of $\mathcal{T}_{\epsilon}$ that corresponds to the discrete groups (Corollary 4.13). This will be carried out in two steps. In the first step we propose an algorithm of Nielsen type [14] which moves any discrete group along its $\mathcal{M}_{\boldsymbol{\epsilon}}$-orbit into $\mathcal{F}_{\boldsymbol{\epsilon}}$. In the second (rather involved) step we show that distinct groups in $\mathcal{F}_{\boldsymbol{\epsilon}}$ have distinct trace spectra and thus are non-conjugate.

AlgoRIthm 4.7. The following algorithm takes as input any $(x, y, z) \in \mathcal{T}_{\boldsymbol{\epsilon}}$ and produces an orbit in $\mathcal{T}_{\boldsymbol{\epsilon}}$.

(i) If $y>z$ execute $\mathbf{y}$-z-symmetry.

(ii) If $z>x y$ execute other $\mathbf{z}$.

(iii) If $x>y z-\boldsymbol{\epsilon} / 4$ execute other $\mathbf{x}$.

If this orbit is discrete, then it is finite and ends in $\mathcal{F}_{\epsilon}$.

Proof. The Euclidean distance from 0 to $(x, y, z)$ in $\mathbb{R}^{3}$ is decreasing $\left(z \geqslant x y \Rightarrow z^{\prime} \leqslant x y\right.$, $\left.x \geqslant y z-(1 / 4) \epsilon \Rightarrow x^{\prime} \leqslant y z-(1 / 4) \epsilon\right)$. Hence, a sequence of these transformations will end in $\mathcal{F}_{\epsilon}$ or accumulate.

THEOREM $4 \cdot 8$.

(i) If $\epsilon=1$ every orbit under the above algorithm is discrete.

(ii) If $\epsilon=-1$ and if any $y<1$ that is encountered is of the form $y=\cos (\pi / n)$ with integer $n$, then the orbit of the algorithm is discrete.

Part (i) is a consequence of the next observation, where we abbreviate

$$
q_{x}= \begin{cases}\frac{1}{2} & \text { if } x \geqslant \frac{5}{4} \\ 2(x-1) & \text { if } 1<x \leqslant \frac{5}{4} .\end{cases}
$$

OBSERVATION 4.9. For $\epsilon=1$, Algorithm 4.7 has the following characteristics.

(a) When other $\mathbf{x}$ is applied, then either $\left|x-x^{\prime}\right| \geqslant 1 / 2$, or the algorithm stops after this step.

(b) When other $\mathbf{z}$ is applied, then either $\left|z-z^{\prime}\right| \geqslant q_{x}$, or the algorithm stops after this step.

Proof. We prove this in a way that makes no use of the geometry of the 2233-groups represented by the triples $(x, y, z)$.

Note that steps (a) and (b) are only applied if $y \leqslant z$.

(a) For given $y, z$ the two roots of $P_{1}(., y, z)$ are of the form $x=y z-1 / 4+r, x^{\prime}=$ $y z-1 / 4-r$, with $r \geqslant 0$, and thus $x^{\prime} \leqslant y z-1 / 4$. If now $r<1 / 4$, then, by the next 
calculation, $z \leqslant x^{\prime} y$, and therefore $\left(x^{\prime}, y, z\right) \in \mathcal{F}_{1}$, which causes the algorithm to halt. The calculation uses Proposition 4.6.

$$
\begin{aligned}
x^{\prime} y-z & =2 y^{2} z-\frac{1}{2} y-x y-z \geqslant 2 y^{2} z-\frac{1}{2} y-y^{2} z-z \\
& =\left(y^{2}-1\right) z-\frac{1}{2} y \geqslant \frac{1}{2 x}\left(\left(2 y^{2}-1\right) z-x y\right) \\
& =\frac{1}{2 x}\left(y(y z-x)+z\left(y^{2}-1\right)\right)>0 .
\end{aligned}
$$

(b) For given $x, y$ the two roots of $P_{1}(x, y,$.$) are of the form z=x y+\rho, z^{\prime}=x y-\rho$ with $\rho \geqslant 0$, and thus $z^{\prime} \leqslant x y$. Now if $\rho<q_{x} / 2$, then, observing that $q_{x} / 2 \leqslant x-1$,

$$
\begin{aligned}
z^{\prime}-y & =(2 x-1) y-z>(2 x-1) y-\left(x y+\frac{1}{2} q_{x}\right) \\
& =y(x-1)-\frac{1}{2} q_{x}>0 .
\end{aligned}
$$

Using Proposition 4.6 again and observing that $z \leqslant x y+q_{x} / 2 \leqslant x y+1 / 4$ we also see that

$$
\begin{aligned}
z^{\prime} y-\frac{1}{4}-x & =2 x y^{2}-y z-\frac{1}{4}-x \geqslant 2 x y^{2}-y\left(x y+\frac{1}{4}\right)-\frac{1}{4}-x \\
& =x\left(y^{2}-1\right)-\frac{1}{4} y-\frac{1}{4} \geqslant \frac{1}{2}+\left(y^{2}-1\right)-\frac{1}{4} y-\frac{1}{4} \\
& =(y-1)\left(y+\frac{3}{4}\right) \\
& >0 .
\end{aligned}
$$

These inequalities yield $\left(x, y, z^{\prime}\right) \in \mathcal{F}_{1}$ and so the algorithm stops.

Since any monotone decreasing sequence of numbers $y=\cos (\pi / n)$ with integer values of $n$ is necessarily finite, the next observation will complete the proof of Theorem $4 \cdot 8$.

OBSERVATION 4.10. For $\epsilon=-1$, Algorithm 4.7 has the following characteristics.

(a) When $y \geqslant 1$ and other $\mathbf{x}$ is applied, then $\left|x-x^{\prime}\right| \geqslant 1 / 2$.

(b) When $y \geqslant 1$ and other $\mathbf{z}$ is applied, then $\left|z-z^{\prime}\right| \geqslant \sqrt{2(x-1)}$.

(c) When $y<1$, then the algorithm produces at most finitely many consecutive other $\mathbf{x}$ and other $\mathbf{z}$ moves.

Proof. (a) Here we have $z \geqslant y \geqslant 1$, and it suffices to observe that the two roots in $x$ of $P_{-1}(x, y, z)=0$ are $y z+1 / 4 \pm(1 / 2) \sqrt{\Delta}$ with $\Delta=4\left(y^{2}-1\right)\left(z^{2}-1\right)+2(y z-1)+1 / 4$.

(b) This is proved in the same way using $(4 \cdot 3)$.

(c) For given $y<1$, the moves may be described by a Chebyshev sequence: set

$$
u=\frac{1}{4\left(1-y^{2}\right)}, \quad v=u-1,
$$

and observe that $u \geqslant 1, v \geqslant 0$ (Definition 4.1). Since $(x+1)\left(1-y^{2}\right) \leqslant 1 / 2$ (Proposition 4.6), we have $1=u-v \leqslant x \leqslant u+v$, and so there exists $\rho>0$ and $\phi \geqslant 0$ such that $y=\cos \rho$ and $x=u-v \cos (\phi)$. If we now define

$$
\begin{aligned}
& x_{k}=u-v \cos (2 k \rho-\phi) \\
& z_{k}=u y-v \cos ((2 k+1) \rho-\phi),
\end{aligned}
$$


then $x_{0}=x$, and we easily check that for any $k$,

$$
P_{-1}\left(x_{k}, y, z_{k-1}\right)=P_{-1}\left(x_{k}, y, z_{k}\right)=P_{-1}\left(x_{k+1}, y, z_{k}\right) .
$$

In particular, $z_{-1}$ and $z_{0}$ are the two roots of $P_{-1}(x, y,)=$.0 , and so either $z=z_{-1}$ or $z=z_{0}$. Therefore, as long as the algorithm does not change $y$, it runs along this sequence. Furthermore, the $x_{k}$ are monotone decreasing. This is only possible for finitely many steps.

It is interesting to watch how the sequence described in (c) operates geometrically. Since $A B C D=-\mathbf{1}$, the products $B C$ and $A D^{-1}$ (of half $\operatorname{trace} \operatorname{tr}(B C)=\operatorname{tr}\left(A D^{-1}\right)=-y$ ) have the same fixed point $p$. The fixed points $a, b, c, d$ of $A, B, C, D$ together with $p$ form two congruent triangles $p b c$ and pad as shown in Figure 1. Using the description given in the proof of Theorem 4.4, we see that other $\mathbf{z}$ simply changes the labels $a, b, c, d$ into $b, a, d, c$, while other $\mathbf{x}$ (used when the labelling is as in the figure) reflects triangle pad along side $p d$ and triangle $p b c$ along side $p b$.

To interpret the above in terms of the Möbius groups we use the following fact about 2-generator Fuchsian groups (see e.g. Rosenberger [13]).

Proposition 4.11. Let $H$ be a group generated by $B, C \in \operatorname{SL}(2, \mathbb{R})$ with $\operatorname{tr}(B)=0$, $\operatorname{tr}(C)=1 / 2$, and set $y=|\operatorname{tr}(B C)|$. If $y \geqslant 1$ then $H$ is always discrete. If $y<1$ then $H$ is discrete if and only if

$$
y=\cos \left(\frac{\pi}{n}\right) \quad \text { for some } \quad n \in \mathbb{N} .
$$

The result is the following, where we restate certain earlier points for convenient reference.

COROLlARY 4-12. Let $\Gamma$ be a 2233-Möbius group.

(i) If $\boldsymbol{\epsilon}=1$, then $\Gamma$ is discrete and is a Fuchsian group of signature $(0 ; 2,2,3,3 ; 0)$. Furthermore, Algorithm 4.7 produces a marking of $\Gamma$ with parameters $(x, y, z) \in \mathcal{F}_{1}$.

(ii) Any $(x, y, z) \in \mathcal{F}_{1}$ is the parameter triple of some Fuchsian group of signature $(0 ; 2,2,3,3 ; 0)$.

(iii) If $\boldsymbol{\epsilon}=-1$ and $\Gamma$ is discrete, then $\Gamma$ is a triangle group of type $(2,3, n)$ with $\infty \geqslant$ $n \geqslant 7$, or the elementary group of order 6 . Furthermore, Algorithm 4.7 produces a marking of $\Gamma$ with parameters $(x, y, z)=(1, y, y) \in \mathcal{F}_{-1}$.

(iv) If $\epsilon=-1$ and $\Gamma$ is not discrete, then Algorithm 4.7 encounters a marking of $\Gamma$ with $y<1$, where $y$ is not of the form $\cos (\pi / n)$ with integer $n$.

In (iii), a $(2,3, \infty)$-group is, by definition, a group generated by elliptic elements $\beta, \gamma$ of order 2 and 3 such that $\beta \gamma$ is parabolic or hyperbolic.

Note that by (iv), Algorithm 4.7 always finds out (not in the numerical sense, of course) whether or not $\Gamma$ is discrete.

Proof. (i) The statement about the signature is Observation $3 \cdot 3$ and Proposition 3.4. By Theorem 4.4 , all points in the orbit represent the same group, and by Theorem 4.8 and the definition of Algorithm 4.7, the final parameters $(x, y, z)$ lie in $\mathcal{F}_{1}$.

(ii) This is part of Proposition $4 \cdot 2$.

(iii) If the group is discrete then the orbit is too and ends in $\mathcal{F}_{-1}$. Hence, the group has a marking with $x=1$, i.e with $\alpha=\beta$ and $\gamma=\delta^{-1}$. The statement thus follows from Proposition $4 \cdot 11$. 
(iv) If the algorithm stops, then it stops at $x=1$, and by Proposition 4.11 we then have $y$ as in the statement. If the algorithm does not stop, then Observation $4 \cdot 10$ tells us that the algorithm produces an infinite decreasing sequence of values $y<1$. But only finitely many of these can have the form $y=\cos (\pi / n)$ with integer $n$.

To complete the discussion of the moduli spaces we note that by (i), $\mathcal{M}_{1}$ moves any point of $\mathcal{T}_{1}$ into $\mathcal{F}_{1}$, and by (ii) and (iii), $\mathcal{M}_{-1}$ moves any point of $\mathcal{T}_{-1}$ belonging to a discrete group, into $\mathcal{F}_{-1}$. The spectral results, Theorem $5 \cdot 1$ for $\epsilon=1$ and Proposition $7 \cdot 1$ for $\epsilon=-1$, will show that the discrete marked groups belonging to $\mathcal{F}_{\epsilon}$ are pairwise non-conjugate in $\operatorname{Isom}(\mathbb{H})$. Together with Theorem 4.4 we have therefore the following.

COROLlary $4 \cdot 13$.

(i) Two groups $\Gamma_{i}$ with parameters $\left(x_{i}, y_{i}, z_{i}\right) \in \mathcal{T}_{\epsilon}$ are conjugate in $\operatorname{Isom}(\mathbb{H})$ if and only if $\left(x_{2}, y_{2}, z_{2}\right)=m\left(x_{1}, y_{1}, z_{1}\right)$ for some $m \in \mathcal{M}_{\boldsymbol{\epsilon}}$.

(ii) The fundamental domain for the action of $\mathcal{M}_{1}$ on $\mathcal{T}_{1}$ is $\mathcal{F}_{1}$.

(iii) The fundamental domain for the action of $\mathcal{M}_{-1}$ on the subset of $\mathcal{T}_{-1}$ corresponding to the discrete groups is

$$
\mathcal{F}_{-1}^{\prime}=\left\{(1, y, y) \mid y=\cos \left(\frac{\pi}{n}\right) \text { for some } n=6,7,8, \ldots, \text { or } y \geqslant 1\right\} \text {. }
$$

\subsection{Inequalities for $\mathcal{F}$}

From now on all considerations concern groups with $\epsilon=1$ and we write for simplicity

$$
P_{1}=P, \quad \mathcal{T}_{1}=\mathcal{T}, \quad \mathcal{F}_{1}=\mathcal{F} .
$$

From the defining inequalities (4.2) and Proposition 4.6 we have on $\mathcal{F}$

$$
\begin{aligned}
& 1<x \leqslant y z-\frac{1}{4} \leqslant x^{\prime} \\
& 1<y \leqslant \quad z \quad \leqslant x y \leqslant z^{\prime} \\
& 1 \leqslant 2(x-1)\left(y^{2}-1\right)
\end{aligned}
$$

where we recall that

$$
x^{\prime}=2 y z-x-\frac{1}{2}, \quad z^{\prime}=2 x y-z .
$$

For $(x, y, z)$ satisfying the inequalities in (4.5), the partial derivatives of $P$ are nonpositive. This implies the following monotonicities in $\mathcal{F}$.

$$
\begin{array}{ll}
\text { For fixed } x: & y \text { increases } \Longleftrightarrow z \text { decreases. } \\
\text { For fixed } y: & z \text { increases } \Longleftrightarrow x \text { decreases. } \\
\text { For fixed } z: & x \text { increases } \Longleftrightarrow y \text { decreases. }
\end{array}
$$

It is helpful to visualize $\mathcal{F}$ by projecting it into the $x y$-plane.

PROPOSITION $4 \cdot 14$. The projection $(x, y, z) \mapsto(x, y)$ yields a bijection of $\mathcal{F}$ onto the set of pairs $(x, y) \in \mathbb{R}^{2}$ satisfying

$$
\begin{aligned}
1+\frac{1}{2\left(y^{2}-1\right)} & \leqslant x \leqslant y+\frac{1}{4(y-1)} \\
1 & <y \leqslant \frac{1}{2} \sqrt{\frac{2 x^{2}+x-1}{x-1}},
\end{aligned}
$$

Proof. For given $(x, y)$ in the image of $\mathcal{F}$ only one of the two roots of $P(x, y, z)=$ 0 is $\leqslant x y$, and so the projection is one-to-one. The root in question is $z=x y-$ 


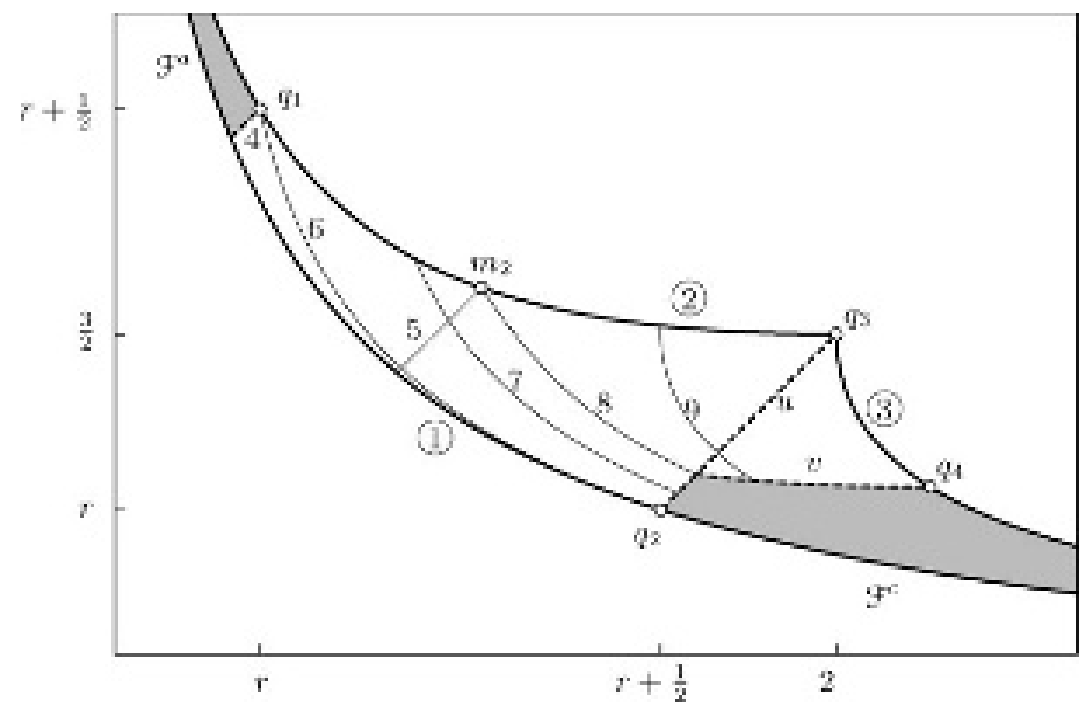

Fig. 2.

$\left(\left(x^{2}-1\right)\left(y^{2}-1\right)-(1 / 2)(x+1)\right)^{\frac{1}{2}}$ and is well defined for any $(x, y)$ satisfying the inequalities on the left-hand side in (4.7). Writing $P=P_{1}$ as in (4.3) respectively as follows,

$$
\begin{aligned}
P(x, y, z)= & -y^{2}(2 x-2)+\frac{1}{2}\left(2 x^{2}+x-1\right)+(z-y)(z+y-2 x y) \\
= & \left(1-\frac{1}{y^{2}}\right)\left(x+y+\frac{1}{4(y+1)}\right)\left(-x+y+\frac{1}{4(y-1)}\right) \\
& +\frac{1}{y^{2}}\left(y z-x-\frac{1}{4}\right)\left(y z+x+\frac{1}{4}-2 x y^{2}\right)
\end{aligned}
$$

and using that $z \leqslant x y$ we see that the inequalities (4.7) are equivalent to the defining inequalities of $\mathcal{F}$, and the lemma follows.

Figure 2 shows the projection of $\mathcal{F}$. Its boundary consists of three smooth arcs, (where in the description we identify $\mathcal{F}$ with its image):

Arc (1) is the curve $2(x-1)\left(y^{2}-1\right)=1$. It is the same (via (4.3)) as $z=x y$ and also the same as $z=z^{\prime}$.

Arc (2) is a truncated part of the curve $y=(1 / 2) \sqrt{\left(2 x^{2}+x-1\right) /(x-1)}$. It is the same (via upper (4.8)) as $y=z$.

Arc (3) is a truncated part of the curve $x=y+1 /(4 y-4)$. It is the same (via lower (4.8)) as $x=y z-1 / 4$.

The dotted lines separate $\mathcal{F}$ into three regions, $\mathcal{F}^{a}, \mathcal{F}^{b}, \mathcal{F}^{c}$ (to be defined in $(5 \cdot 1)$ ), which will need different spectral considerations. Line $y=x+1 / 2$, (4) meets side $y=z$ at point $q_{1}=(r, r+1 / 2)$, where

$$
r=\frac{1+\sqrt{17}}{4} .
$$

Line $x=y+1 / 2,(u)$ goes from point $q_{2}=(r+1 / 2, r)$ on side $z=z^{\prime}$ to the common vertex $q_{3}=(2,3 / 2)$ of the two opposite sides. Line $z=y+1 / 2,(v)$ meets side $x=x^{\prime}$ at $q_{4}=((2+\sqrt{5}) / 2,(3+\sqrt{5}) / 4)$. 
As additional information (not used explicitly in the sequel) we have plotted lines 4 9 showing loci of equality between certain traces, namely, $4: y=x+1 / 2,5: y=x, 6$ : $z=x+1 / 2,7: x^{\prime}=z^{\prime}, 8: x=z, 9: x^{\prime}=x+1 / 2$. Lines $u$ and $v$ do not represent loci of equality between traces.

Point $m_{2}$ on arc (2) satisfies $x=y=z$ and corresponds to the second example described in Section 8. The first example, $m_{1}$ lies on arc (1) with $x=1.0574 \ldots$ and is outside the scope of the figure.

The next lemma gathers relations which will be used to distinguish certain spectra from each other.

LEMMA 4·15. For $(x, y, z) \in \mathcal{F}$ the following hold:

(i) $x^{\prime}, z^{\prime} \geqslant x, y, z$;

(ii) $y>x+1 / 2 \Longrightarrow x<(1+\sqrt{17}) / 4$;

(iii) $x=y+1 / 2 \Longrightarrow y \geqslant(1+\sqrt{17}) / 4$;

(iv) $z=y+1 / 2 \Longrightarrow y \geqslant(3+\sqrt{5}) / 4$;

(v) $x=z+1 / 2 \Longrightarrow y=z=3 / 2, x=x^{\prime}=2$.

Proof. (i) By (4.5) we have $z^{\prime} \geqslant x y>x, y$ and $z^{\prime} \geqslant z, x^{\prime} \geqslant x$. Now assume that $x^{\prime} \leqslant z$, i.e. $2 y z-x-1 / 2 \leqslant z$. Then $x \leqslant z$ and $2 y z-2 z \leqslant x-z+1 / 2 \leqslant 1 / 2$, from which we obtain $(y-1) x \leqslant(y-1) z \leqslant 1 / 4$. On the other hand, $\left(y^{2}-1\right)(x-1) \geqslant 1 / 2$, and therefore $(y-1) x \geqslant\left(y^{2}-1 / 2\right) /(y+1)$. It follows that $0 \geqslant 4\left(y^{2}-1 / 2\right)-(y+1)=(y-1)(4 y+3)$, a contradiction. Hence, $x^{\prime} \geqslant z$. Since $z \geqslant y$, this establishes (i).

(ii) By Lemma $4 \cdot 14$ we have $4 y^{2}(x-1) \leqslant 2 x^{2}+x-1$. The largest possible $x$ (for $(x, y, z) \in \mathcal{F})$ for which this can hold simultaneously with $y \geqslant x+1 / 2$, is the larger solution to $2 x^{2}-x-2=0$. This yields (ii).

(iii) Use that $2(x-1)\left(y^{2}-1\right) \geqslant 1(4 \cdot 5)$.

(iv) On line $z=y+1 / 2$ in $\mathcal{F}, y$ is a monotone decreasing function of $x$ (4.6). The minimum is reached on the boundary of $\mathcal{F}$, that is, when $x=y+1 /(4 y-4)=y z-1 / 4=$ $y(y+1 / 2)-1 / 4$. Solving this equation for $y$ we get the bound.

(v) By (4.5) and the current hypothesis, we have $1<y z-1 / 4-x=(y-1) x-1 / 4-$ $(1 / 2) y$, and therefore $(1 / 2) y \leqslant(y-1) x-1 / 4$. Using that $P(x, y, z)=P(x, y, x-1 / 2)=$ 0 , we obtain $(1 / 2) y(2 x-1) \leqslant(2 x-1)((y-1) x-1 / 4)=y^{2}$. Hence, $z=x-1 / 2 \leqslant y$. But $z \geqslant y$, and therefore $z=x-1 / 2=y$. Using $0=P(x, x-1 / 2, x-1 / 2)=-x(2 x-1)(x-2)$ we conclude that $x=2$.

For the inequalities in the next lemma we denote by $x_{y}$ the minimal value that $x$ can assume in $\mathcal{F}$ when $y$ is given. Similarly, $y_{x}$ denotes the minimal value $y$ can assume in $\mathcal{F}$ when $x$ is given. By (4.7), these values are

$$
x_{y}=\frac{2 y^{2}-1}{2 y^{2}-2}, \quad y_{x}=\sqrt{\frac{2 x-1}{2 x-2}} .
$$

LEMMA 4.16. For any $(x, y, z) \in \mathcal{F}$ we have:

(i) $z \leqslant x y_{x}, z \leqslant y x_{y}$;

(ii) $(x-1)(y-1) \leqslant 1 / 2$;

(iii) If $x \geqslant y+1 / 2$, then $z \leqslant x+1 / 2$ and $x, z<2 x_{y}^{2}-1$;

(iv) If $\min \{x, z\} \leqslant y+1 / 2$, then $x+1 / 2, z \leqslant 2 y<2 y^{2}-1 / 2,4 y_{x}^{3}-3 y_{x}$.

Proof. (i) This follows from the monotonicities (4.6) and by applying the inequality $z \leqslant$ $x y(4.5)$ to the extremal cases. 
(ii) If $x \leqslant 2$, then by (4.7), $y \leqslant \frac{1}{2} \sqrt{\frac{(x+1)(2 x-1)}{x-1}} \leqslant \frac{2 x-1}{2 x-2}$ and the inequality follows. If $x>2$ then $y<\frac{3}{2}$ and, also by (4.7), $x \leqslant y+\frac{1}{4(y-1)} \leqslant \frac{2 y-1}{2 y-2}$, which again yields the inequality. Note that we have equality at $x=2, y=\frac{3}{2}$.

(iii) Here $\left(x-\frac{1}{2}\right)^{2} \geqslant y^{2} \geqslant y_{x}^{2}=\frac{2 x-1}{2 x-2}$. By (i) we have $z^{2} \leqslant x^{2} y_{x}^{2}=x^{2} \frac{2 x-1}{2 x-2}$. Plugging this into the identity

$$
\left(x+\frac{1}{2}\right)^{2}-x^{2} \frac{2 x-1}{2 x-2}=\frac{1}{2 x-1}\left(\left(x-\frac{1}{2}\right)^{2}-\frac{2 x-1}{2 x-2}\right)
$$

we obtain $z \leqslant x+1 / 2$. Since $x \geqslant y+1 / 2$ we have $y \leqslant 3 / 2$ and $x_{y} \geqslant x_{3 / 2}=7 / 5$. Hence, by $(4 \cdot 7)$,

$$
x \leqslant y+\frac{1}{4(y-1)}=\frac{y+1}{2}\left(x_{y}+1\right)-1 \leqslant \frac{5}{4}\left(x_{y}+1\right)-1<2 x_{y}^{2}-1 .
$$

For the second inequality we use that $x_{y}$ and $x_{y}\left(2 x_{y}-3 / 2\right)$ are monotone decreasing functions of $y$ for $y>1$. For $y=(3 / 2)$ we have $x_{y}\left(2 x_{y}-3 / 2\right)>1$. Together with (i) we obtain $z \leqslant y x_{y} \leqslant(3 / 2) x_{y}<2 x_{y}^{2}-1$.

(iv) We begin with the first two inequalities on the left-hand side of " $2 y$ ", where we first consider the case $x \leqslant y+1 / 2$. Then $x+1 / 2<x+y-1 / 2 \leqslant 2 y$. The condition $x \leqslant y+1 / 2$ implies that $x \leqslant 2$ and therefore by $(4 \cdot 5), z \leqslant x y \leqslant 2 y$.

Now consider the case $x \geqslant y+1 / 2, z \leqslant y+1 / 2$. Then, of course, $z<2 y$. The condition $x \geqslant y+1 / 2$ implies that $y \leqslant 3 / 2$. If we increase $x$ keeping $y$ fixed then $z$ decreases. By $(4 \cdot 7)$ and (4.5), the maximal value of $x$ is reached when

$$
x=y+\frac{y}{4(y-1)}, \quad x=y z-\frac{1}{4} .
$$

It suffices therefore to prove the inequality $x+1 / 2 \leqslant 2 y$ under this special hypothesis. If we further increase $x$, but now under this hypothesis, then $y$ decreases and so $z$ increases. The extremal case is reached when $z=y+1 / 2$, and in this case we obtain the equations $x=y(y+1 / 2)-1 / 4=y+1 /(4 y-4)$. The solutions to these with $x, y>1$ are $y=$ $(3+\sqrt{5}) / 4, x=1+\sqrt{5} / 2$ (vertex $q_{4}$ in Figure 2 ). Hence, in the extremal case we have $x+1 / 2=2 y$, and the inequality follows.

For the proof of the two inequalities on the right hand side of " $2 y$ " in (iv) we first observe that for any $(x, y, z) \in \mathcal{F}$ satisfying the hypothesis of (iv) we have $x \leqslant 1+\sqrt{5} / 2$ (equality at $\left.q_{4}\right)$ and $y \geqslant(1+\sqrt{17}) / 4$ (equality at $\left.q_{2}\right)$.

In fact, if $x \geqslant y+1 / 2$ and $z \leqslant y+1 / 2$, then by what we have just shown, the largest possible value of $x$ is $1+\sqrt{5} / 2$ and the smallest possible value of $y$ is $(3+\sqrt{5}) / 4$. If $x \leqslant y+1 / 2$, then the largest possible value of $x$ is 2 , and the smallest possible value of $y$ is obtained when $x=y+1 / 2=\left(2 y^{2}-1\right) /\left(2 y^{2}-2\right)(4 \cdot 7)$, that is, for $y=(1+\sqrt{17}) / 4$.

For the inequality $2 y<2 y^{2}-1 / 2$ it suffices now to observe that the zeros of the polynomial $2 y^{2}-2 y-1 / 2$ are smaller than $(1+\sqrt{17}) / 4$.

For the final inequality we use that $y_{x}$ and hence the expression $\left(y_{x}-1\right)\left(4 y_{x}^{2}-3\right)+2 y_{x}^{2}$ is a monotone decreasing function of $x$ and larger than 3 for $x \leqslant 1+\sqrt{5} / 2$. Hence, $y_{x}\left(4 y_{x}^{2}-3\right)>$ $2 y_{x}^{2}=2+1 /(x-1) \geqslant 2 y$ by (ii). 


\section{The bottom of the spectrum and spectral rigidity}

In this section we prove the spectral rigidity of the 2233-Möbius groups with $\epsilon=1$ or, what is the same (Corollary 4.12(i)), the Fuchsian groups of signature $(0 ; 2,2,3,3 ; 0)$. The remaining, rather simple, part of the proof of the main Theorem 1.3 is postponed to Section 7. By Corollary 4.12, up to conjugation in $\operatorname{Isom}(\mathbb{H})$, any Fuchsian group of signature $(0 ; 2,2,3,3 ; 0)$ is of the form $G / \pm 1$, where $G=G(x, y, z)$ is a marked 2233-matrix group with parameters $(x, y, z) \in \mathcal{F}$. Conversely, any $(x, y, z) \in \mathcal{F}$ belongs to such a group.

For the spectra we use the following notation. For $X \in G$ we have the class $c(X)$ of all $Y \in G$ which are conjugate to $\pm X$ in $G$, and the extended class $c^{\prime}(X)=c(X) \cup c\left(X^{-1}\right)$. We denote by $P(G)$ (respectively $P^{\prime}(G)$ ) the set of all $c(X)$ (respectively $c^{\prime}(X)$ ), where $X$ is a hyperbolic primitive element of $G$, i.e. $X$ is none of the elliptic elements of order 2 and 3 in $G$, and $X$ is not of the form $Y^{n}$ for some $Y \in G$ and $n \geqslant 2$. With this we consider the following spectra:

$$
\Pi_{G}=\{|\operatorname{tr}(X)| \mid c(X) \in P(G)\} \quad \text { and } \quad \Lambda_{G}=\left\{|\operatorname{tr}(X)| \mid c^{\prime}(X) \in P^{\prime}(G)\right\} .
$$

The members of $\Pi_{G}$ and $\Lambda_{G}$ will be called the (spectral) "lines". A moment's reflection shows that for 2233-matrix groups $G_{1}, G_{2}$ we have

$$
\begin{aligned}
& \Pi_{G_{1}}=\Pi_{G_{2}} \Longleftrightarrow \mathrm{TS}\left(G_{1} / \pm 1\right)=\mathrm{TS}\left(G_{2} / \pm 1\right) \\
& \Lambda_{G_{1}}=\Lambda_{G_{2}} \Longleftrightarrow \operatorname{TS}^{\prime}\left(G_{1} / \pm 1\right)=\operatorname{TS}^{\prime}\left(G_{2} / \pm 1\right) .
\end{aligned}
$$

The goal of this section is to prove the following, where " $G \in \mathcal{F}$ " is short hand for " $G=$ $G(x, y, z)$ with $(x, y, z) \in \mathcal{F}$ '”

THEOREM 5·1. For $G_{1}, G_{2} \in \mathcal{F}$ the following implications hold:

$$
\begin{aligned}
& \Pi_{G_{1}}=\Pi_{G_{2}} \Longrightarrow G_{1}=G_{2}, \\
& \Lambda_{G_{1}}=\Lambda_{G_{2}} \Longrightarrow G_{1}=G_{2} .
\end{aligned}
$$

We will prove the theorem for $\Lambda_{G}$ and add the necessary modifications for $\Pi_{G}$ at the end of this section.

The next two theorems will allow us to work with a very restricted part of the spectrum. In these theorems, $\Lambda_{X Y}$, for $X, Y \in G$, denotes the subsequence of all those lines in $\Lambda_{G}$ whose extended class can be represented by a member of the subgroup $H_{X Y}$ generated by $X, Y$. In a joint sequence like $\Lambda_{X Y} \cup \Lambda_{X^{\prime} Y^{\prime}}$, a line that occurs in $\Lambda_{X Y}$ and $\Lambda_{X^{\prime} Y^{\prime}}$ is listed only once. The proofs of the two theorems are postponed to Section 6 .

THEOREM 5.2. For any $(x, y, z) \in \mathcal{F}$ the following statements hold:

(i) the first line in $\Lambda_{G}$ is either $x$ or $y$;

(ii) the first two lines in $\Lambda_{C D}$ are $x$ and $x+1 / 2$; the third line in $\Lambda_{C D}$ is larger than $x+1 / 2$

(iii) the first two lines in $\Lambda_{A D} \cup \Lambda_{B C}$ are $y$ and $2 y^{2}-1 / 2$.

For the second theorem we introduce the following subsets of $\mathcal{F}$ (shaded areas in Figure 2),

$$
\begin{aligned}
& \mathcal{F}^{a}=\left\{(x, y, z) \in \mathcal{F} \mid y>x+\frac{1}{2}\right\}, \\
& \mathcal{F}^{c}=\left\{(x, y, z) \in \mathcal{F} \mid x, z>y+\frac{1}{2}\right\}, \\
& \mathcal{F}^{b}=\mathcal{F} \backslash\left(\mathcal{F}^{a} \cup \mathcal{F}^{c}\right) .
\end{aligned}
$$


THEOREM 5·3. Any $G=G(x, y, z) \in \mathcal{F}$ has the following properties:

(a) if $G \in \mathcal{F}^{a}$, then the first line in $\Lambda_{G} \backslash \Lambda_{C D}$ is $y$;

(b) if $G \in \mathcal{F}^{b}$, then the first four lines in $\Lambda_{G}$ are among $x, y, z, x+1 / 2, x^{\prime}, z^{\prime}$;

(c) if $G \in \mathcal{F}^{c}$, then the first two lines in $\Lambda_{G} \backslash\left(\Lambda_{A D} \cup \Lambda_{B C}\right)$ are $x$ and $z$.

We use this to characterize the sets $\mathcal{F}^{a}, \mathscr{F}^{b}, \mathcal{F}^{c}$ by an initial part of the spectrum.

LEMMA 5.4. Let $(x, y, z) \in \mathcal{F}$ and denote by $\ell_{1}, \ell_{2}, \ell_{3}$ with $\ell_{1} \leqslant \ell_{2} \leqslant \ell_{3}$ the values of the first three lines in the spectrum of $G(x, y, z)$. Then the following hold.

$$
\begin{aligned}
&(x, y, z) \in \mathcal{F}^{a} \Longleftrightarrow \ell_{1}<\frac{1+\sqrt{17}}{4} \text { and } \ell_{1}+\frac{1}{2}=\ell_{2}<\ell_{3}, \\
&(x, y, z) \in \mathcal{F}^{c} \Longleftrightarrow \ell_{1}+\frac{1}{2}<\ell_{2} .
\end{aligned}
$$

Proof. Let us first take $G \in \mathcal{F}^{a}$. By Theorem 5-2(ii), the first two lines in $\Lambda_{C D}$ are $x$ and $x+1 / 2$, and the third line in $\Lambda_{C D}$ is larger than $x+1 / 2$. By Theorem 5.3(a), the first line outside $\Lambda_{C D}$ is $y$, where by hypothesis $y>x+1 / 2$. This shows that $\ell_{3}>\ell_{2}=\ell_{1}+1 / 2$. The upper bound for $\ell_{1}$ stems from Lemma 4.15(ii).

Now take $G \in \mathcal{F}^{b}$. Then $y \leqslant x+1 / 2$. In the case $x \leqslant y$ we have by Theorem 5.2(i) that $\ell_{1}=x$, and it follows that $\ell_{2} \leqslant y \leqslant x+1 / 2=\ell_{1}+1 / 2$ with equality only if $y=x+1 / 2$, in which case we have $\ell_{2}=\ell_{3}=\ell_{1}+1 / 2$. In the case $x \geqslant y$ we have again by Theorem 5.2(i) that $\ell_{1}=y$. Since $G \notin \mathcal{F}^{c}$, either $x$ or $z$ is $\leqslant y+1 / 2$, and therefore $\ell_{2} \leqslant \ell_{1}+1 / 2$. Together with Lemma 4.15(iii)(iv), this shows that for $G \in \mathcal{F}^{b}$ we have $\ell_{2} \leqslant \ell_{1}+1 / 2$ and equality can only occur if either $\ell_{2}=\ell_{3}$ or if $\ell_{1} \geqslant(1+\sqrt{17}) / 4$.

Finally, take $G \in \mathcal{F}^{c}$. Then $\ell_{1}=y$. By Theorem 5.2(iii), the first two lines in $\Lambda_{A D} \cup \Lambda_{B C}$ are $y$ and $2 y^{2}-1 / 2>y+1 / 2$. By Theorem 5.3(c), the first line outside $\Lambda_{A D} \cup \Lambda_{B C}$ is $x$ or $z$ and both are larger than $y+1 / 2$. This completes the proof of the lemma.

Let us now take $\left(x_{1}, y_{1}, z_{1}\right),\left(x_{2}, y_{2}, z_{2}\right) \in \mathcal{F}$ and compare the groups

$$
G_{1}=\left(x_{1}, y_{1}, z_{1}\right), \quad G_{2}=\left(x_{2}, y_{2}, z_{2}\right)
$$

under various spectral hypotheses. By Lemma 5.4, a first result is this:

LEMMA 5.5. If the first three lines of $\Lambda_{G_{1}}$ and $\Lambda_{G_{2}}$ coincide, then only three cases are possible: either $G_{1}, G_{2} \in \mathcal{F}^{a}$ or $G_{1}, G_{2} \in \mathcal{F}^{b}$ or $G_{1}, G_{2} \in \mathcal{F}^{c}$.

LEMMA 5.6. If $\left\{x_{1}, y_{1}, z_{1}\right\}=\left\{x_{2}, y_{2}, z_{2}\right\}$, then $G_{1}=G_{2}$.

Proof. $0=P\left(x_{1}, y_{1}, z_{1}\right)-P\left(x_{2}, y_{2}, z_{2}\right)=\left(x_{1}-x_{2}\right) / 2$ implies $x_{1}=x_{2}$, and we have $y_{1} \leqslant z_{1}, y_{2} \leqslant z_{2}$.

LEMMA 5.7. If $G_{1}, G_{2} \in \mathcal{F}^{a}$ and the lines $\leqslant y_{1}$ in $\Lambda_{G_{1}}$ and $\Lambda_{G_{2}}$ coincide, then $G_{1}=G_{2}$.

Proof. By Theorem 5·2(i), $\ell_{1}=x_{1}=x_{2}$. Since on $\mathcal{F}$ the traces of $H_{C D}$ are functions that depend only on $x$, the parts $\Lambda_{C D}$ in the spectra of $G_{1}$ and $G_{2}$ coincide. Consequently, the first line outside $\Lambda_{C D}$ is also the same for $G_{1}$ and $G_{2}$. Since for any group $G$ the first line in $\Lambda_{C D}$ is $x$ (Theorem 5.2) and the first line outside is $y$ (Theorem 5.3) we obtain $x_{1}=x_{2}$ and $y_{1}=y_{2}$.

LEMMA 5.8. If $G_{1}, G_{2} \in \mathcal{F}^{c}$ and the lines $\leqslant \max \left\{x_{1}, z_{1}\right\}$ in $\Lambda_{G_{1}}$ and $\Lambda_{G_{2}}$ coincide, then $G_{1}=G_{2}$. 
Proof. By Theorem 5.2(i) we have $y_{1}=y_{2}$. As in the previous case we conclude that for $G_{1}$ and $G_{2}$ the parts $\Lambda_{A D} \cup \Lambda_{B C}$ of the spectra coincide. Using Theorem 5.3(c) we obtain $\left\{x_{1}, z_{1}\right\}=\left\{x_{2}, z_{2}\right\}$, and Lemma 5.6 implies $G_{1}=G_{2}$.

The remaining case to consider is $G_{1}, G_{2} \in \mathcal{F}^{b}$. Here we shall work with the set of lines

$$
\left\{x, y, z, x+\frac{1}{2}, x^{\prime}, z^{\prime}\right\} \text {. }
$$

For any $G=G(x, y, z)$ we call the sequence of these lines arranged in increasing order the string of $G$. To make the ordering unique we apply the notational convention that if two lines are equal we use lexicographic order w.r.t the symbol list (5.2). Thus, in a statement like "part of the string is $y, z, x$ " we read the additional information that $z$ is strictly smaller than $x$.

Even though the remaining case only concerns couples in $\mathcal{F}^{b}$, everything that follows (up to Proposition 5.13) is true for any pair $G_{1}, G_{2} \in \mathcal{F}$.

We first make the following general observation:

OBSERVATION 5.9. For any $G \in \mathcal{F}$, the first three lines in the string are in one of the following orders:

$\begin{array}{rccc}\text { (i) } & x & y & z \\ \text { (ii) } & x & x+\frac{1}{2} & y \\ \text { (iii) } & x & y & x+\frac{1}{2}\end{array}$

$\begin{array}{ccc}y & x & x+\frac{1}{2} \\ y & z & x \\ y & x & z\end{array}$

Proof. Lemma $4 \cdot 15$ shows that $x^{\prime}$ and $z^{\prime}$ do not occur. For the remaining symbols any order not in this list violates (4.7).

We apply this to $G_{1}$ and $G_{2}$ with the respective strings $\left\{x_{1}, y_{1}, z_{1}, x_{1}+1 / 2, x_{1}^{\prime}, z_{1}^{\prime}\right\}$ and $\left\{x_{2}, y_{2}, z_{2}, x_{2}+1 / 2, x_{2}^{\prime}, z_{2}^{\prime}\right\}$. For either string the initial triple is in one of the six possible orders. The next lemma shows that only few combinations are possible.

LEMmA 5.10. If $G_{1} \neq G_{2}$ and the first three lines in the strings of $G_{1}$ and $G_{2}$ coincide, then the orders of the triples are in one of the following combinations: (ii)-(iv), (ii)-(vi), (iii)-(v).

Proof. In column (i),(ii),(iii) of Observation 5.9 the pairings (i)-(i), (i)-(iii), (ii)-(ii), (iii)(iii) imply $x_{1}=x_{2}, y_{1},=y_{2}$ and are therefore excluded. (i)-(ii) cannot occur because in (i) we have $z \leqslant x+1 / 2$, while in (ii) we have $y>x+1 / 2$, by the notational convention. For the same reason, (ii)-(iii) is impossible.

In column (iv),(v),(vi) the pairings (iv)-(iv), (iv)-(vi), (v)-(v), (vi)-(vi) again imply $G_{1}=$ $G_{2}$. Pairing (iv)-(v), say $G_{1}$ with (iv) and $G_{2}$ with (v), implies $x_{2}=z_{2}+1 / 2$ so that by Lemma 4.15(v) we have $y_{2}=z_{2}$ and therefore $y_{1}=x_{1}$, contrary to the notational convention. Pairing (v)-(vi) is excluded by Lemma 5.6.

As for the combinations across the columns, (i)-(iv), say $G_{1}$ with (i) and $G_{2}$ with (iv), is impossible because then $z_{1} \leqslant x_{1}+1 / 2$ while $x_{2}+1 / 2>y_{2}+1 / 2$. Lemma 5.6 further excludes (i)-(v), (i)-(vi), and Lemma 5·11, below, excludes (ii)-(v), (iii)-(vi). Finally, (iii)(iv) is impossible because it implies $x_{1}=y_{2}<x_{2}=x_{1}$.

LEMMA 5.11. If $G_{1}, G_{2} \in \mathcal{F}$ and $x_{1}=y_{2}<x_{2}=y_{1}$, then $z_{1}<z_{2}$. 
Proof. By (4.5) we have $z_{1}, z_{2} \leqslant x_{1} y_{1}=x_{2} y_{2}$. Furthermore, $0=P\left(x_{2}, y_{2}, z_{2}\right)-$ $P\left(x_{1}, y_{1}, z_{1}\right)=z_{2}^{2}-z_{1}^{2}-2 x_{1} y_{1}\left(z_{2}-z_{1}\right)+(1 / 2)\left(x_{2}-x_{1}\right)=\left(z_{2}-z_{1}\right)\left(z_{2}+z_{1}-2 x_{1} y_{1}\right)+$ $(1 / 2)\left(x_{2}-x_{1}\right)$. Now $(1 / 2)\left(x_{2}-x_{1}\right)>0$ and $z_{1}+z_{2}-2 x_{1} y_{1} \leqslant 0$, hence $z_{2}-z_{1}>0$.

To exclude the remaining cases we have to take a fourth spectral line into account.

OBSERVATION 5·12. If $G_{1} \neq G_{2}$ and the first four lines in the strings coincide, then, possibly after interchanging $G_{1}$ and $G_{2}$, these lines occur in one of the following pairs of orders:

$\begin{array}{cccccccccc}\text { (ii.iv) } & x_{1} & x_{1}+\frac{1}{2} & y_{1} & z_{1} & & & & & \\ & y_{2} & x_{2} & x_{2}+\frac{1}{2} & z_{2} & & & & & \\ \text { (iii.v.i) } & x_{1} & y_{1} & x_{1}+\frac{1}{2} & z_{1} & \text { (ii.vi.i) } & x_{1} & x_{1}+\frac{1}{2} & y_{1} & z_{1} \\ & y_{2} & z_{2} & x_{2} & x_{2}+\frac{1}{2} & & y_{2} & x_{2} & z_{2} & x_{2}+\frac{1}{2} \\ \text { (iii.v.ii) } & x_{1} & y_{1} & x_{1}+\frac{1}{2} & z_{1} & \text { (ii.vi.ii) } & x_{1} & x_{1}+\frac{1}{2} & y_{1} & z_{1} \\ & y_{2} & z_{2} & x_{2} & x_{2}^{\prime} & & y_{2} & x_{2} & z_{2} & x_{2}^{\prime} \\ & & & & & & & & & \\ \text { (iii.v.iii) } & x_{1} & y_{1} & x_{1}+\frac{1}{2} & z_{1} & \text { (ii.vi.iii) } & x_{1} & x_{1}+\frac{1}{2} & y_{1} & z_{1} \\ & y_{2} & z_{2} & x_{2} & z_{2}^{\prime} & & y_{2} & x_{2} & z_{2} & z_{2}^{\prime}\end{array}$

Proof. If $x+1 / 2$ is among the first four lines in the string of $G \in \mathcal{F}$, then $x^{\prime}$ and $z^{\prime}$ cannot occur (Lemma 4·15(i)). Hence, in Observation 5·9.(ii),(iii) and(iv), the fourth line has to be $z$. Otherwise the fourth line is among $\left\{x+1 / 2, x^{\prime}, z^{\prime}\right\}$. All other cases have already been excluded by Lemma $5 \cdot 10$.

Now we exclude these cases as well.

Exclusion of combination (ii.iv): Here we have $0=P\left(x_{1}, y_{1}, z_{1}\right)-P\left(x_{2}, y_{2}, z_{2}\right)=$ $P\left(y_{2}, x_{2}+1 / 2, z_{2}\right)-P\left(x_{2}, y_{2}, z_{2}\right)=P\left(y_{2}, y_{2}+1, z_{2}\right)-P\left(y_{2}+1 / 2, y_{2}, z_{2}\right)=y_{2}+1 / 2-y_{2} z_{2}$, hence $z_{2}=1+1 /\left(2 y_{2}\right)$. This implies $z_{2}<1+y_{2}=x_{2}+1 / 2$, but $z_{2} \geqslant x_{2}+1 / 2$.

Exclusion of (iii.v.i) and (ii.vi.i): Here $0=P\left(x_{1}, y_{1}, z_{1}\right)-P\left(x_{2}, y_{2}, z_{2}\right)=P\left(y_{2}, z_{2}, y_{2}+\right.$ 1) $-P\left(y_{2}+1 / 2, y_{2}, z_{2}\right)=y_{2}+1 / 2-y_{2} z_{2}$, hence $z_{2}=1+1 /\left(2 y_{2}\right)$. This implies $0=$ $P\left(y_{2}+1 / 2, y_{2}, 1+1 /\left(2 y_{2}\right)\right)=-\left(2 y_{2}^{3}-2 y_{2}^{2}-4 y_{2}-1\right) /\left(2 y_{2}\right)^{2}$ and therefore $0=\left(2 y_{2}^{3}-\right.$ $\left.2 y_{2}^{2}-4 y_{2}-1\right)=-2 y_{2}^{2}\left(z_{2}-y_{2}\right)-3 y_{2}-1<0$, a contradiction.

Exclusion of (iii.v.ii) and (ii.vi.ii): In these cases $0=P\left(x_{1}, y_{1}, z_{1}\right)-P\left(x_{2}^{\prime}, y_{2}, z_{2}\right)=$ $P\left(y_{2}, z_{2}, x_{2}^{\prime}\right)-P\left(x_{2}^{\prime}, y_{2}, z_{2}\right)=(1 / 2)\left(y_{2}-x_{2}^{\prime}\right)$, hence $x_{2}^{\prime}=y_{2}=z_{2}=x_{2}=y_{2}+1 / 2$, impossible.

Exclusion of (iii.v.iii) and (ii.vi.iii): Observe that we have $x_{2}=y_{2}+1 / 2$. We will show, using (4.5), that then $x_{2}+1 / 2 \leqslant z_{2}^{\prime}$ :

In fact, $z_{2}^{\prime}-\left(x_{2}+1 / 2\right) \geqslant x_{2} y_{2}-\left(x_{2}+1 / 2\right)=\left(y_{2}+1 / 2\right) y_{2}-\left(y_{2}+1\right)=y_{2}^{2}-(1 / 2) y_{2}-1$. On the other hand we have $0 \leqslant\left(x_{2}^{2}-1\right)\left(y_{2}^{2}-1\right)-(1 / 2)\left(x_{2}+1\right)$. This yields with $x_{2}=y_{2}+1 / 2$ : $y_{2}\left(2 y_{2}+3\right)\left(y_{2}^{2}-(1 / 2) y_{2}-1\right) / 2 \geqslant 0$. Hence we get $y_{2}^{2}-(1 / 2) y_{2}-1 \geqslant 0$.

Since none of the cases in Observation 5.12 is possible, we arrive at the following result.

PROPOSITION 5.13. If for two 2233-matrix groups (with $\epsilon=1$ ) the smallest four lines among $\left\{x, y, z, x+1 / 2, x^{\prime}, z^{\prime}\right\}$ coincide, then these groups are conjugate. 
With this proposition, the proof of Theorem $5 \cdot 1$ for $\Lambda_{G}$ is complete: Lemmata $5 \cdot 5,5 \cdot 7$ and 5.8 show that non-trivial isospectral pairs $G_{1}, G_{2}$ in $\mathcal{F}$ can only occur in $\mathcal{F}^{b}$, but for $G_{1}, G_{2} \in \mathcal{F}^{b}$, Theorem $5 \cdot 3$ and Proposition, 5.13 say that if the first four lines in the spectra coincide, then $G_{1}=G_{2}$.

The proof of Theorem $5 \cdot 1$ for $\Pi_{G}$ goes along the same lines, with the reasoning made simpler due to the slightly different multiplicities.

We observe that for $X \in G$ the (non-extended) class contains $X^{-1}$ if and only if $X$ is of the form $X=Y_{1} Y_{2}$, where $Y_{1}, Y_{2}$ are of order two. Thus, e.g. $x$ counts as one line in $\Pi_{G}$, whereas $y, z, x+1 / 2$ each counts as two lines.

By Theorem 5.2, the first line $\ell_{1}^{\prime}$ in $\Pi_{G}$ is still either $x$ or $y$. By Lemma 5.4 the initial part $\ell_{1}^{\prime}, \ell_{2}^{\prime}, \ell_{3}^{\prime}, \ell_{4}^{\prime}$ (in increasing order) of $\Pi_{G}$ looks therefore as follows.

$$
\begin{aligned}
& G \in \mathcal{F}^{a} \Longrightarrow \ell_{1}^{\prime}=x, \ell_{2}^{\prime}=\ell_{3}^{\prime}=x+\frac{1}{2}, \ell_{4}^{\prime}>x+\frac{1}{2} ; \\
& G \in \mathcal{F}^{c} \Longrightarrow \ell_{1}^{\prime}=\ell_{2}^{\prime}=y, \ell_{3}^{\prime}>y+\frac{1}{2} \\
& G \in \mathcal{F}^{b} \& x \leqslant y \Longrightarrow \ell_{1}^{\prime}=x, l_{4}^{\prime} \leqslant x+\frac{1}{2} \\
& G \in \mathcal{F}^{b} \& x>y \Longrightarrow \ell_{1}^{\prime}=\ell_{2}^{\prime}=y, \ell_{3}^{\prime}<y+\frac{1}{2} .
\end{aligned}
$$

This shows that for $G_{1}, G_{2} \in \mathcal{F}$, Lemma 5.5 holds also with respect to $\Pi_{G_{1}}$ and $\Pi_{G_{2}}$, if we spell it out for the first four lines.

The proof of Lemma 5.7 goes through without modifications, and so there are no isospectral pairs in $\mathcal{F}^{a}$. The same holds for Lemma 5.8 and $\mathcal{F}^{c}$.

For the remaining possible pairs in $\mathcal{F}^{b}$, we remark using Theorem 5·3(b) and Observation 5.9 that for $G \in \mathcal{F}^{b}$ the first five elements of $\Pi_{G}$ are in one of the following orders.

$$
\begin{array}{ll}
\ell_{1}^{\prime}=x \leqslant \ell_{2}^{\prime}=\ell_{3}^{\prime}=y \leqslant \ell_{4}^{\prime}=\ell_{5}^{\prime}=z, & \ell_{1}^{\prime}=\ell_{2}^{\prime}=y<\ell_{3}^{\prime}=x<\ell_{4}^{\prime}=\ell_{5}^{\prime}=x+\frac{1}{2} \\
\ell_{1}^{\prime}=x<\ell_{2}^{\prime}=\ell_{3}^{\prime}=x+\frac{1}{2}<\ell_{4}^{\prime}=\ell_{5}^{\prime}=y, & \ell_{1}^{\prime}=\ell_{2}^{\prime}=y \leqslant \ell_{3}^{\prime}=\ell_{4}^{\prime}=z<\ell_{5}^{\prime}=x \\
\ell_{1}^{\prime}=x \leqslant \ell_{2}^{\prime}=\ell_{3}^{\prime}=y \leqslant \ell_{4}^{\prime}=\ell_{5}^{\prime}=x+\frac{1}{2}, & \ell_{1}^{\prime}=\ell_{2}^{\prime}=y<\ell_{3}^{\prime}=x \leqslant \ell_{4}^{\prime}=\ell_{5}^{\prime}=z .
\end{array}
$$

The argument used in the proof of Lemma $5 \cdot 10$ shows that for $G_{1} \neq G_{2}$ no pairing of orderings in the same column is possible. But pairings across the columns are not possible either, because in the left column the multiplicity of $\ell_{1}^{\prime}$ is either 1 or 3 or $\geqslant 5$ while in the right column it is either 2 or 4 . This completes the proof of Theorem $5 \cdot 1$.

\section{Geometric estimates}

This section contains the proofs of Theorems $5 \cdot 2$ and $5 \cdot 3$.

We first observe that 5.2(i) is a consequence of the remaining parts of the two theorems: For $G \in \mathcal{F}^{a}$ it is implied by 5·2(ii) and 5.3(a), for $G \in \mathcal{F}^{c}$ by $5 \cdot 2$ (iii) and 5·3(c) (because $y \leqslant z(4 \cdot 5)$ ), and for $G \in \mathcal{F}^{b}$ by $5 \cdot 3$ (b) and Lemma $4 \cdot 15(\mathrm{i})$.

For the proof of Theorem 5.2(ii) we deal with the group $H_{C D}$ generated by $C, D$, using Figure 3. In this figure, $a, b, c, d$ are the fixed points of $A, B, C, D$, respectively, and $\gamma$ is the axis of $A B=(C D)^{-1}$. The geodesics $\gamma$ and $D(\gamma)$ are separated by the geodesic through $c, d$ and have positive distance.

Now consider the right angled geodesic hexagons $H_{d}, H_{c}$ with centers $c$ and $d$, bounded by $\gamma, D(\gamma), D^{-1}(\gamma)$ and the common perpendiculars between these geodesics, respectively by $\gamma, C(\gamma), C^{-1}(\gamma)=D(\gamma)$ and their common perpendiculars. The sides of a hexagon on $\gamma, C(\gamma)$, etc. are called sides of type $\gamma$, the remaining sides are called sides of type $\beta$.

We denote by $x, \eta$ and $\sigma$ the hyperbolic cosines (cosh) of the lengths of, respectively, a side of type $\gamma$, a side of type $\beta$ and the perpendicular from a side of type $\gamma$ to an opposite 


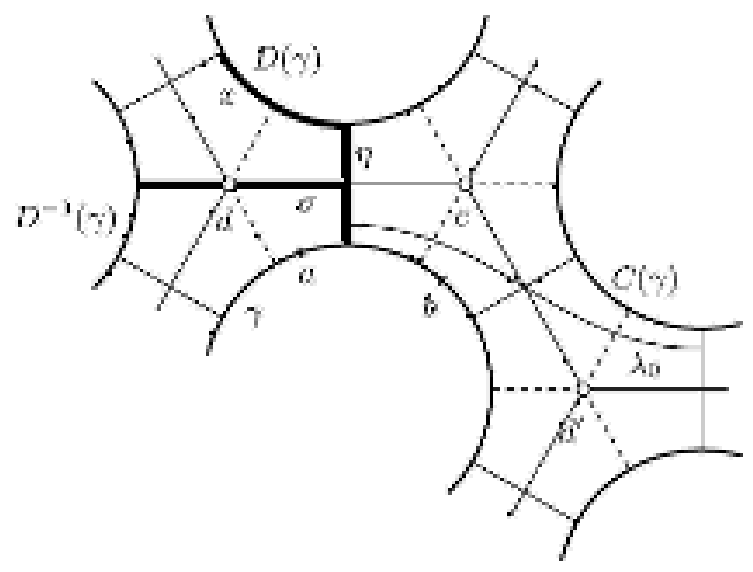

Fig. 3.

side of type $\beta$ (an altitude of the hexagon). Observe that indeed, $x=-\operatorname{tr} C D$. The quantity $\sigma$ may also be characterized as follows

$$
\sigma=\cosh \frac{1}{2} \operatorname{dist}\left(D^{-1}(\gamma), C(\gamma)\right) .
$$

From the trigonometry of hyperbolic polygons we have the following relations.

$$
\begin{aligned}
(x-1)(\eta-1) & =1, \\
\sigma^{2}=(x+1)(\eta+1) & =\frac{2 x^{2}}{x-1}+1=\frac{2 \eta^{2}}{\eta-1}+1 .
\end{aligned}
$$

(To get $(6 \cdot 1)$, apply [7, formula $2 \cdot 3 \cdot 1(i)]$ to any of the six quadrilaterals built by the altitudes of $H_{d}$; to get (6.2), apply [7, formula 2.3.4(i)] to one of the two pentagons into which $H_{d}$ is decomposed by an altitude.)

We let $T$ be the subset of the hyperbolic plane $\mathbb{H}$ covered by the images of $H_{d}$ and $H_{c}$ under the group $H_{C D}$. Likewise, $T$ may be obtained by successively reflecting $H_{d}$ across sides of type $\beta$. The images of $T$ under the action of $G$ cover $\mathbb{H}$ without overlapping, hence a tiling of $\mathbb{H}$ with hexagons that is invariant under the action of $G$. Part of this tiling is shown schematically in Figure 4.

We use the following terminology. If $g \in G$ is hyperbolic, then a geodesic $\operatorname{arc} \zeta$ in $\mathbb{H}$ is called a fundamental arc for $g$, if $\zeta$ lies on the axis $\alpha_{g}$ of $g$ and is a fundamental domain for the action of the cyclic group $\langle g\rangle$ on $\alpha_{g}$. The length $\ell(\zeta)$ satisfies

$$
\cosh \frac{1}{2} \ell(\zeta)=|\operatorname{tr} g|
$$

We shall also say, more generally, that $\zeta$ is a fundamental arc for $g$ if it is a fundamental arc for some $\tilde{g}$ in the conjugacy class of $g$.

Any hyperbolic element in $H_{C D}$ can be represented in its extended conjugacy class by some word

$$
W=C^{\varepsilon_{1}} D^{\delta_{1}} \cdots C^{\varepsilon_{n}} D^{\delta_{n}}
$$

where all $\varepsilon_{i}, \delta_{i} \in\{-1,1\}$. For the words with two letters we have the following traces,

$$
x=-\operatorname{tr} C D, \quad x+\frac{1}{2}=\operatorname{tr} C D^{-1} .
$$

Any other line in the spectrum $\Lambda_{C D}$ of $H_{C D}$ corresponds to a word $W$ with at least four 
letters, where we claim that

$$
|\operatorname{tr} W| \geqslant 2 x^{2}+x-\frac{1}{2}
$$

For the proof we use that $W$ has a fundamental $\operatorname{arc} \zeta \subset T$ that crosses $2 n$ hexagons having its end points on sides of type $\beta$. Since $W$ is primitive, not all exponents have the same value and, accordingly, there exists an arc $\lambda \subset \zeta$ that crosses a pair of hexagons in such a way that it connects opposite sides like arc $\lambda_{0}$ in $H_{c} \cup H_{d^{\prime}}$ as shown in Figure 3. In this figure $\lambda_{0}$ is meant to be the shortest connection between the two sides in question, and we have $\ell(\lambda) \geqslant \ell\left(\lambda_{0}\right)$. Together with the perpendicular from $\gamma$ to $C(\gamma)$ it forms a crossed right angled geodesic hexagon. By [7, formula 2.4.4] and using $(6 \cdot 1)$, we obtain $\cosh \lambda_{0}=$ $\left(x^{2}-1\right) \eta+x^{2}=2 x^{2}+x$.

If two non-overlapping arcs such as $\lambda$ occur in $\zeta$, then $\operatorname{tr} W \geqslant 2 x^{2}+x$. If only one such arc occurs then, changing $W$ in its extended conjugacy class, if necessary, we may assume that $W=C^{\varepsilon_{1}} D^{-\varepsilon_{1}}(C D)^{n-1}$. The traces $t_{n-1}$ in this case satisfy

$$
t_{0}=x+\frac{1}{2}, \quad t_{1}=-\left(2 x^{2}+x-\frac{1}{2}\right), \quad t_{n}=-2 x t_{n-1}-t_{n-2}, \quad n=2,3, \ldots
$$

(use 2.2(i)), and their absolute values are monotone increasing with $n$. This proves (6.4) and hence point (ii) in Theorem $5 \cdot 2$.

Point (iii) will be proved in a different way and is postponed to Section 7 (Proposition $7 \cdot 1)$.

For the proof of Theorem $5 \cdot 3$ we make the following definition. Two boundary components $\gamma^{\prime}, \gamma^{\prime \prime}$ of $T$ are called neighbours (to each other) if there exists a hexagon of the tiling of $T$ with a side on $\gamma^{\prime}$ and another side on $\gamma^{\prime \prime}$. For example, $D^{-1}(\gamma)$ and $D(\gamma)$ are neighbours, but $D^{-1}(\gamma)$ and $C(\gamma)$ are not.

LEMMA 6.1. If two boundary components $\gamma^{\prime}, \gamma^{\prime \prime}$ of $T$ are not neighbours then we have $\cosh \left(\operatorname{dist}\left(\gamma^{\prime}, \gamma^{\prime \prime}\right) / 2\right) \geqslant \sigma$.

Proof. Consider the domains $S^{\prime}, S^{\prime \prime}$ formed by all the hexagons of the tiling that have a side on $\gamma^{\prime}$, respectively on $\gamma^{\prime \prime}$. By hypothesis, $S^{\prime}$ and $S^{\prime \prime}$ do not overlap. Any curve from $\gamma^{\prime}$ to $\gamma^{\prime \prime}$ in $T$ is therefore at least as long as twice the altitude of a hexagon.

LEMMA 6.2. For any $(x, y, z) \in \mathcal{F}$ we have $\sigma \geqslant 2 y$. If $\min \{x, z\} \leqslant y+1 / 2$, then $\sigma \geqslant x, y, x+1 / 2, z$.

Proof. The first inequality follows from (6.2) and (4.7). If $\min \{x, z\} \leqslant y+1 / 2$ then by Lemma $4 \cdot 16$ (iv), $2 y \geqslant x+1 / 2, z$, hence the remaining inequalities.

Let now $g \in G$ be a primitive hyperbolic element. For the proof of Theorem $5 \cdot 3$ we first deal with points (a) and (b). There are four cases to consider.

Case 1: $g$ is conjugate to an element in $H_{C D}$.

For (a) there is nothing to prove. For (b) we may assume $g \in H_{C D}$. If $g$ is conjugate to $C D$, $C D^{-1}$ or their inverses, then $|\operatorname{tr} g|$ is line $x$ or $x+1 / 2$. Otherwise, $|\operatorname{tr} g| \geqslant 2 x^{2}+x-1 / 2>$ $x(x+1 / 2) \geqslant x y \geqslant z$ (by (6.4) and (4.5)), and $|\operatorname{tr} g|$ is not among the first four lines. This settles the case.

In the remaining cases the axis of $g$ is not contained in $T$ nor in any image of $T$ under the action of $G$. Since these images fill out $\mathbb{H}$, it follows that $g$ has a fundamental arc with an end point on the boundary of $T$. 


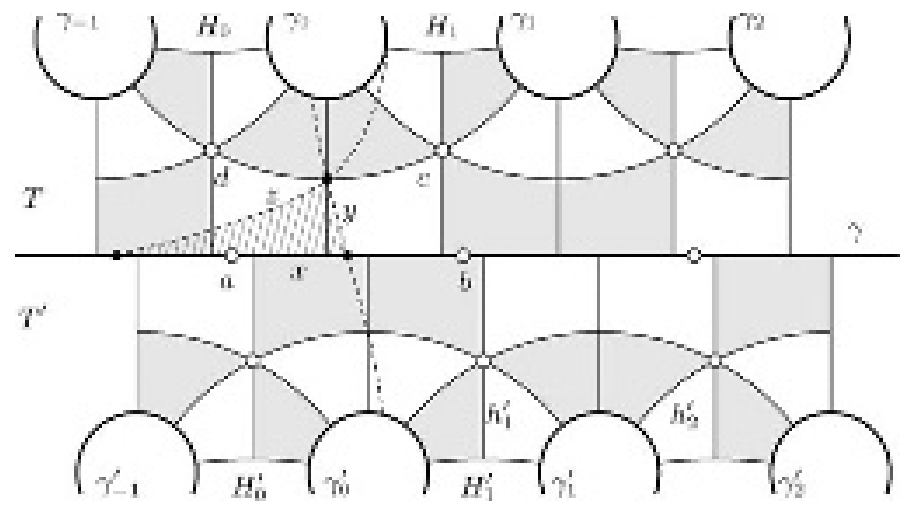

Fig. 4.

Case 2: $g$ has a fundamental arc $\zeta$ with both end points on the boundary of $T$.

By Lemma 6.1 and 6.2 we may assume that $\zeta$ connects neighbours. We may further assume that $\zeta$ lies on the axis of $g$ itself, and, using conjugation with a suitable element in $H_{C D}$, that the initial point of $\zeta$ lies on $C^{-1}(\gamma)$. Since $g$ sends this point to the endpoint of $\zeta$ on $\gamma$, we have $g\left(C^{-1}(\gamma)\right)=\gamma$. Now $B C$ also sends $C^{-1}(\gamma)$ to $\gamma$, and we have therefore $g(B C)^{-1}(\gamma)=\gamma$ and $g(B C)^{-1}(T)=T$. Since the maximal subgroup of $G$ that preserves $\gamma$ and $T$ is the cyclic group generated by $A B$, we conclude that for some $n \in \mathbb{Z}, g=$ $(A B)^{n} B C$.

LEMMA 6.3. Set $\tau_{n}=\operatorname{tr}(A B)^{n} B C$. The function $n \mapsto\left|\tau_{n}\right|, n \in \mathbb{Z}$, is convex with a minimum at $n=0$. It is strictly decreasing in the range $n \leqslant 0$ and strictly increasing in the range $n \geqslant 1$.

Proof. Recall thattr $B C=-y$. By 2·2(i),

$$
\tau_{n+1}=-2 x \tau_{n}-\tau_{n-1} .
$$

For $n=0,1,-1$ the values are $\tau_{0}=-y, \tau_{1}=z, \tau_{-1}=2 x y-z=z^{\prime}$. By (4.5), $y \leqslant$ $z \leqslant x y \leqslant z^{\prime}$. Since $x>1$, we conclude that the signs of the $\tau_{n}$ are alternating and, hence, $\left|\tau_{n-1}\right|+\left|\tau_{n+1}\right|=2 x\left|\tau_{n}\right|>2\left|\tau_{n}\right|$. The lemma follows.

For $n=2$ we compute $\left|\tau_{2}\right|=2 x z-y \geqslant 2 x y-z=z^{\prime}$. By Lemma 4.15(i), $z^{\prime} \geqslant x, y, z$. From Lemma $6 \cdot 3$ we now obtain that if $n \neq-1,0,1$, then $|\operatorname{tr} g| \geqslant \max \left\{x, y, z, z^{\prime}\right\}$. This settles the proof of Theorem 5·3(a)(b) in Case 2.

Case 3: $g$ has a fundamental arc $\zeta$ with end points on the boundary of $T \cup A(T)$. Here it will turn out that we only need to know the shortest element.

Let $T^{\prime}=A(T)$ and denote by $\gamma_{k}, \gamma_{k}^{\prime}, k \in \mathbb{Z}$, the boundary components of $T$, respectively $T^{\prime}$, as shown in Figure 4, where the labeling is such that

$$
\gamma_{0}=(B C)^{-1}(\gamma), \quad \gamma_{0}^{\prime}=B C(\gamma), \quad \gamma_{1}^{\prime}=(C B)^{-1}(\gamma), \quad \gamma_{1}=C B(\gamma),
$$

and such that for any $j \in \mathbb{Z}, A B\left(\gamma_{j}\right)=\gamma_{j-2}$ and $A B\left(\gamma_{j}^{\prime}\right)=\gamma_{j-2}^{\prime}$.

By Lemma 6.1 and 6.2 we have to consider only the case where in $T$ and $T^{\prime}$ arc $\zeta$ connects neighbours. Furthermore, we may assume that $\zeta$ lies on the axis of $g$ itself and that for some $m \in \mathbb{Z}$ it goes from either $\gamma_{2 m}$ or $\gamma_{2 m+1}$ to either $\gamma_{0}^{\prime}$ or $\gamma_{1}^{\prime}$. Therefore, $g$ has the form $g=B C^{\delta}(A B)^{k} B C^{\varepsilon}(A B)^{m}$, with $k, m \in \mathbb{Z}$ and $\delta, \varepsilon \in\{-1,1\}$. (If, e.g. $\delta=\varepsilon=1$, then 
$(B C)^{-1} g$ and $B C(A B)^{m}$ both send $\gamma_{2 m}$ to $\gamma$ and $T$ to $T^{\prime}$, which is only possible if $(B C)^{-1} g$ and $B C(A B)^{m}$ differ by an element of $\langle A B\rangle$, etc.) Changing $g$ in its equivalence class once more, if necessary, we finally may assume that $g$ has the following form, with $k, m \in \mathbb{Z}$,

$$
g=B C^{ \pm 1}(A B)^{k} B C(A B)^{m} .
$$

LEMMA 6.4. Let $\delta= \pm 1, g_{l m}=B C^{\delta}(A B)^{\delta l} B C(A B)^{m}, l, m \in \mathbb{Z}$, and set $t_{l m}=\left|\operatorname{tr} g_{l m}\right|$. For any $l$, the function $m \mapsto t_{l m}, m \in \mathbb{Z}$, is convex, strictly decreasing in the range $m \leqslant 0$, and strictly increasing in the range $m \geqslant 1$. The same statement holds for the function $l \mapsto t_{l m}$ for any given $m$.

Proof. Convexity in $m$ for any fixed $l$ and vice versa follows from 2.2(i) by the same argument as in the proof of Lemma 6.3. To determine the minima we use a geometric consideration.

For $k \in \mathbb{Z}$ we denote, respectively, by $H_{k}, H_{k}^{\prime}$ the hexagons of the tilings of $T, T^{\prime}$ whose sides are on $\gamma$ and $\gamma_{k-1}, \gamma_{k}$, respectively $\gamma_{k-1}^{\prime}, \gamma_{k}^{\prime}$. By $h_{k}, h_{k}^{\prime}$ we denote the altitudes of these hexagons standing on $\gamma$. Finally we let $\xi$ be the length of any side of type $\gamma$, so that $x=\cosh \xi$.

$H_{0}$ is shifted towards $H_{-1}^{\prime}$ by some amount $s$ that lies between 0 and $\xi$. To see this, we consider the axes of $B C$ and $A C$ (dotted lines in Figure 4). They pass through the midpoint of segment $d c$ and through the centers of symmetry of $H_{1} \cup H_{0}^{\prime}$, respectively $H_{0} \cup H_{-1}^{\prime}$. Together with $\gamma$ they form a geodesic triangle with hyperbolic cosines of sides equal to $x, y, z$. If we increase $y$ (in $\mathcal{F}$ ) keeping $x$ fixed, then $z$ decreases, by (4.6). We also know by (4.5) that $y \leqslant z$. Therefore, the shift of $H_{0}$ is in the direction of $H_{-1}^{\prime}$, and the amount $s$ lies between 0 and $\xi$.

We prove the lemma for the case $\delta=-1$. For $\delta=+1$ the proof is similar. Thus, let $\zeta$ be the fundamental arc of $g_{l m}$ going from an initial point $p \in \gamma_{2 m}$ to the endpoint $q=g_{l m}(p) \in$ $\gamma_{1}^{\prime}$. We let $\bar{p}, \bar{q} \in \gamma$ be the orthogonal projections of $p, q$ upon $\gamma$. The points $p, \bar{p}, \bar{q}, q$ form a self crossing geodesic quadrilateral, and by [7, formula 2.3.2] we have

$$
\cosh \ell(\zeta)=u \cosh (\bar{p} \bar{q})+v
$$

where $\bar{p} \bar{q}$ is the distance from $\bar{p}$ to $\bar{q}$, and $u=\cosh (p \bar{p}) \cosh (q \bar{q}), v=\sinh (p \bar{p}) \sinh (q \bar{q})$.

If $m \leqslant-1$, we set $p_{*}=(A B)^{-1}(p), \bar{p}_{*}=(A B)^{-1}(\bar{p})$. Then $g_{l, m+1}\left(p_{*}\right)=g_{l m}(p)=q$. Since $p \bar{p}$ lies between the altitudes $h_{2 m}, h_{2 m+1}$, and $q \bar{q}$ between $h_{1}^{\prime}, h_{2}^{\prime}$, and since $H_{0}$ is shifted towards $H_{-1}^{\prime}$, we have $\bar{p} \bar{q}=2|m| \xi+\rho$, for some $\rho>0$, and $\bar{p}_{*} \bar{q}=(2|m|-1) \xi+\rho<$ $\bar{p} \bar{q}$. By the above formula we also have $p_{*} q<p q$. Since for any hyperbolic transformation the distance from a point to its image becomes minimal if the point lies on the axis of the transformation, we conclude that $t_{l, m+1} \leqslant \cosh \left((1 / 2) p_{*} q\right)<\cosh ((1 / 2) p q)=t_{l m}$.

If $m \geqslant 2$, we set $p_{*}=(A B)(p), \bar{p}_{*}=(A B)(\bar{p})$ and get similarly, $t_{l, m-1}<t_{l m}$. (In the special case $m=2$, the inequality $\bar{p}_{*} \bar{q}<\bar{p} \bar{q}$ is deduced from the fact that $\bar{p} \bar{q}>2 \xi-s$ and $\bar{p}_{*} \bar{q}<2 \xi-s$.)

This proves the claimed monotonicities in $m$, for any given $l$. To prove the statement with the roles of $l$ and $m$ reversed, we observe that $B C^{-1}(A B)^{-l} B C(A B)^{m}$ is conjugate to $B C(A B)^{m} B C^{-1}(A B)^{-1}$ and apply the same procedure to the latter. 
By Lemma 6.4, the shortest element in (6.5) is among those with $0 \leqslant l, m \leqslant 1$. Here we get five primitive equivalence classes. They are represented by the following:

$$
\begin{aligned}
& g_{1}=B C B C A B \\
& g_{2}=B C^{-1} B C \\
& g_{3}=B C^{-1} B C A B \\
& g_{4}=B C^{-1}(A B)^{-1} B C \\
& g_{5}=B C^{-1}(A B)^{-1} B C A B .
\end{aligned}
$$

We show that no absolute trace for this list is smaller than $y$, and if $\min \{x, z\} \leqslant y+1 / 2$, then none goes below the forth line in $\Lambda_{G}$. This will settle the proof of Theorem $5 \cdot 3(\mathrm{a})(\mathrm{b})$ in Case 3. The traces are computed via 2.2(i), and we obtain the following:

$$
\begin{aligned}
\left|\operatorname{tr} g_{3}\right| & =-\operatorname{tr} B C A C^{-1}=2 y z-x-\frac{1}{2}=x^{\prime} \geqslant x, y, z, \\
\left|\operatorname{tr} g_{1}\right| & =\operatorname{tr} A C B C=2 \operatorname{tr} A C \operatorname{tr} B C-\operatorname{tr} A B^{-1}=2 y z-x=x^{\prime}+\frac{1}{2}, \\
\left|\operatorname{tr} g_{4}\right| & =-\operatorname{tr} A B C B(B C)^{-1}=-2 \operatorname{tr} A B C B \operatorname{tr} B C+\operatorname{tr} A B C B^{2} C \\
& =2 y z^{\prime}-x-\frac{1}{2} \geqslant x^{\prime}, \\
\left|\operatorname{tr} g_{2}\right| & =-\operatorname{tr} C B C^{-1} B=-2 \operatorname{tr} C B \operatorname{tr} C^{-1} B+\operatorname{tr} C^{2}=2 y^{2}-\frac{1}{2}>y, \\
\left|\operatorname{tr} g_{5}\right| & =-\operatorname{tr} A B C A(B C)^{-1}=-\operatorname{tr} D A D^{-1} A=-\operatorname{tr} C B C^{-1} B=\left|\operatorname{tr} g_{2}\right| .
\end{aligned}
$$

In the first line, $\left|\operatorname{tr} g_{3}\right|$ is $x^{\prime}$, and the inequality is from Lemma 4.15(i). Now $\left|\operatorname{tr} g_{1}\right| \geqslant y$, $\left|\operatorname{tr} g_{4}\right| \geqslant y$, and none of the two can get below the fourth line in $\Lambda_{G}$. The same holds for $\left|\operatorname{tr} g_{2}\right|$ and $\left|\operatorname{tr} g_{5}\right|$ because if $\min \{x, z\} \leqslant y+1 / 2$, then by Lemma 4.16(iv), $2 y^{2}-1 / 2 \geqslant$ $2 y \geqslant x, y, z, x+1 / 2$.

Case 4: $g$ has a fundamental $\operatorname{arc} \zeta$ that crosses at least three copies of $T$.

Set $r=(1 / 2) \operatorname{dist}\left(\gamma, \gamma_{0}\right)$. Then by $(6 \cdot 1)$ and the definition of $\eta$ (see Figure 3) we have $\cosh (2 r)=\eta=1+1 /(x-1)=2 y_{x}^{2}-1(4 \cdot 9)$, that is, $y_{x}=\cosh r$. Since $(1 / 2) \ell(\zeta) \geqslant 3 r$, we obtain

$$
|\operatorname{tr} g| \geqslant \cosh (3 r)=4 y_{x}^{3}-3 y_{x}>\eta \text {. }
$$

By Lemma $4 \cdot 16$ (ii) we have $\eta=1+1 /(x-1) \geqslant 2 y-1>y$. This settles (a) in Theorem 5.3. If $\min \{x, z\} \leqslant y+1 / 2$, then by Lemma 4.16(iv), $4 y_{x}^{3}-3 y_{x} \geqslant x, y, z, x+1 / 2$. This settles (b).

The proofs of (a) and (b) in Theorem 5.3 are now complete. For the proof of (c) we use similar arguments but with the roles of $A B$ and $B C$ reversed (albeit the new situation is not entirely symmetric to the old one).

Thus, let $\beta$ be the axis of $B C=(D A)^{-1}$ and consider the hexagons $\mathcal{H}_{c}, \mathcal{H}_{d}^{\prime}$ built by $\beta$, $\beta_{0}:=C(\beta), \beta_{1}:=C^{-1}(\beta)$ (and the common perpendiculars), respectively $\beta, \beta_{1}^{\prime}:=D(\beta)$, $\beta_{0}^{\prime}:=D^{-1}(\beta)$, (Figure 5 ). The images of $\mathcal{H}_{c}$ under $H_{B C}$ tile a simply connected domain $\mathcal{T}$ and the images of $\mathcal{H}_{d}^{\prime}$ under $H_{A D}$ tile a similar domain $\mathcal{T}^{\prime}$ adjacent to $\mathcal{T}$ along $\beta$. The images of $\mathcal{T} \cup \mathcal{T}^{\prime}$ under $G$ tile $\mathbb{H}$, but in contrast to the case studied before, there exists no element in $G$ that sends $\mathcal{T}$ to $\mathcal{T}^{\prime}$. We denote by $\beta_{k}=(B C)^{-k}\left(\beta_{0}\right), \beta_{k}^{\prime}=(B C)^{-k}\left(\beta_{0}^{\prime}\right)$ the neighbours of $\beta$ on $\mathcal{T}$ and $\mathcal{T}^{\prime}$.

If $g \in G$ is not conjugate to any element in $H_{B C}$ or $H_{A D}$, then $g$ has a fundamental arc $\zeta$ on its axis that crosses a number of copies (under $G$ ) of $\mathcal{T} \cup \mathcal{T}^{\prime}$, and we first consider the 


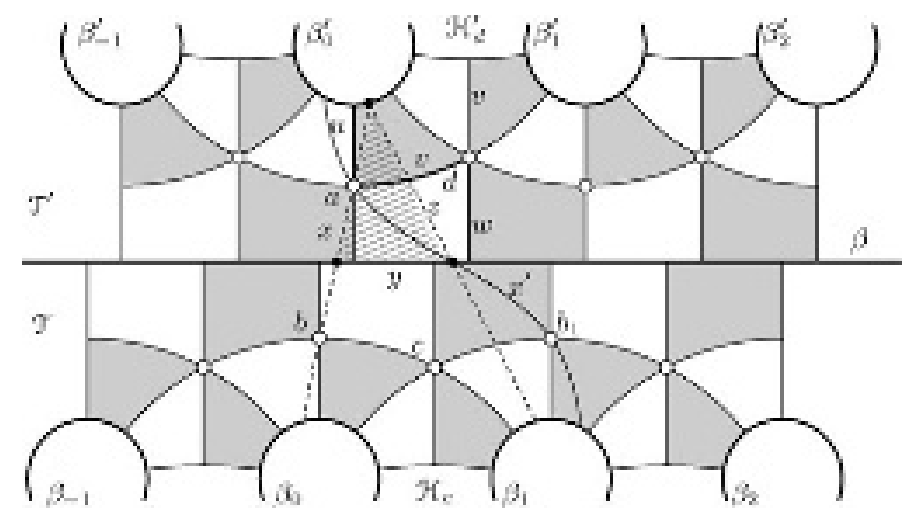

Fig. 5.

case where $\zeta$ crosses exactly one such copy and, moreover, such that in $\mathcal{T}$ as well as $\mathcal{T}^{\prime}$ it connects neighbours. Here $g$ is conjugate to one of the following $h_{l m}$, where $l, m \in \mathbb{Z}$,

$$
h_{l m}=A(B C)^{l} B(B C)^{m} .
$$

Note that $h_{l m}$ has a fundamental arc going from $\beta_{m}$ to $\beta_{0}^{\prime}$. Figure 5 shows these arcs for $h_{00}=A B, h_{01}=-A C$ and $h_{-11}=A\left(C^{-1} B C\right)$. The axes of $A B$ and $A\left(C^{-1} B C\right)$ pass through $b$ and $a$, respectively $b_{1}$ and $a$, where $b_{1}=C^{-1}(b)$ is the fixed point of $C^{-1} B C$. Note also that $x=|\operatorname{tr} A B|=\cosh \operatorname{dist}(a, b)$ and $x^{\prime}=\left|\operatorname{tr} A\left(C^{-1} B C\right)\right|=\cosh \operatorname{dist}\left(a, b_{1}\right)$.

If in $\mathcal{F}$ we increase $x$ keeping $y$ fixed, then $x^{\prime}$ decreases and we always have $x \leqslant x^{\prime}$. (Recall that $x^{\prime}=2 y z-x-1 / 2$ and $z$ decreases by (4.6).) Hence, $\mathcal{H}_{c}$ is shifted towards $B C\left(\mathcal{H}_{d}^{\prime}\right)$ by an amount that lies between 0 and half the side of $\mathcal{H}_{c}$ on $\beta$. Using this we prove in the same way as Lemma 6.4 the following.

LEMMA 6.5. For any $l \in \mathbb{Z}$ the function $m \mapsto \tau_{l m}=\left|\operatorname{tr} h_{l m}\right|, m \in \mathbb{Z}$, is convex. It is strictly decreasing in the range $m \leqslant-1$ and strictly increasing in the range $m \geqslant 1$. The same statement holds for the function $l \mapsto \tau_{l m}$ for any given $m$.

Using 2.2(i) we compute the following table. (Observe that for any $l, m \in \mathbb{Z}, h_{l m}$ and $h_{-m,-l}$ lie in the same extended conjugacy class. A convenient order of computation is this: $\tau_{00}=x, \tau_{-1,0}=\tau_{01}=z, \tau_{10}=\tau_{0,-1}=z^{\prime}, \tau_{-1,-1}=\tau_{11}=x+1 / 2, \tau_{-1,1}=x^{\prime}$, $\left.\tau_{1,-1}=2 y z^{\prime}-(x+1 / 2) \geqslant 2 y z-x-1 / 2=x^{\prime}, \tau_{-2,0}=\tau_{02}=2 y z-x=x^{\prime}+1 / 2.\right)$

$$
\begin{aligned}
& \tau_{02}=x^{\prime}+\frac{1}{2} \\
& \tau_{-1,1}=x^{\prime} \quad \tau_{01}=z \quad \tau_{11}=x+\frac{1}{2} \\
& \tau_{-2,0}=x^{\prime}+\frac{1}{2} \quad \tau_{-1,0}=z \quad \tau_{00}=x \quad \tau_{10}=z^{\prime} \\
& \tau_{-1,-1}=x+\frac{1}{2} \quad \tau_{0,-1}=z^{\prime} \quad \tau_{1,-1} \geqslant x^{\prime} .
\end{aligned}
$$

By Lemma 4.15(i), $x^{\prime}, z^{\prime} \geqslant \max \{x, z\}$. Since we assume $x \geqslant y+1 / 2$ (because $G \in \mathcal{F}^{c}$ ), it follows from Lemma $4 \cdot 16$ (iii) that $x+1 / 2 \geqslant z$. Hence, the $\tau_{l m}$ computed in this table are either equal to $x$ or $z$ ore else bounded below by $\max \{x, z\}$. Using Lemma 6.5 we conclude that the first two lines coming from the list (6.6) are $x$ and $z$.

For the proof of Theorem 5.3(c), two cases remain: the case where $g$ has a fundamental $\operatorname{arc} \zeta$ that does not connect neighbours, and the case where $\zeta$ crosses at least two copies of $\mathcal{T} \cup \mathcal{T}^{\prime}$. For the first case let $u$ be the perpendicular geodesic segment from $a$ to $\beta_{0}^{\prime}, v$ the segment $a d$ and $w$ the perpendicular segment from $d$ to $\beta$. By $u, v, w$, we also denote the 
lengths of these segments. Note that $u$ is also the length of the shortest connection from $v$ to $\beta$, so that $u<w$.

For fixed $y$, the maximal possible value of $z$ (for $(x, y, z) \in \mathcal{F}$ ), is reached when $x$ is minimal. In this extremal case, the axis of $A C$ passes through the end point of $u$ on $\beta_{0}^{\prime}$ and the end point of $w$ on $\beta$, as may be checked considering the images of these points under $C^{-1} A$. Therefore, $z<\cosh (u+v+w)$. In a similar way we see that $x<\cosh (y / 2+$ $2 u+y / 2)<\cosh (v+u+w)$. Assuming, for simplicity, that $\zeta$ crosses $\mathcal{T} \cup \mathcal{T}^{\prime}$ but does not connect neighbours on $\mathcal{T}^{\prime}$, we have that the length of $\zeta \cap \mathcal{T}^{\prime}$ is at least two altitudes of a hexagon, that is, at least $2 w+2 v$. Since $u<w$, the part $\zeta \cap \mathcal{T}$ has length $\geqslant 2 u$. Altogether, $|\operatorname{tr} g|=\cosh (\ell(\zeta) / 2) \geqslant \cosh (u+v+w)>x, z$, which settles the case.

Let us finally consider the case where $g$ has a fundamental arc that crosses two or more copies of $\mathcal{T} \cup \mathcal{T}^{\prime}$. By what has just been shown, we may assume that $\zeta$ only connects neighbours. For fixed $y$, the minimal possible value of $x$ for $(x, y, z) \in \mathcal{F}$ is $x_{y}=\left(2 y^{2}-1\right) /\left(2 y^{2}-\right.$ 2) (4.9). Since $\cosh (\ell(\zeta) / 2) \geqslant \cosh \left(2 \operatorname{dist}\left(\beta_{0}^{\prime}, \beta\right)\right)$, where $\cosh \left(\operatorname{dist}\left(\beta_{0}^{\prime}, \beta\right)\right)=x_{y}$, we have $|\operatorname{tr} g| \geqslant 2 x_{y}^{2}-1$. By Lemma $4 \cdot 16$ (iii), $2 x_{y}^{2}-1 \geqslant x, z$.

The proof of Theorem $5 \cdot 3$ is now complete.

\section{On the spectrum of $H_{B C}$}

The following arguments are due to Binotto [2]. They allow us to compute an initial part of the trace spectrum of $H_{B C}$ and thus fill in the missing part (iii) in the proof of Theorem 5.2.

Here too the signs of the traces play an active role. The main tool is Lemma 2.2(i) which we restate in the following form,

$$
\begin{aligned}
\operatorname{tr} X Y+\operatorname{tr} X Y^{-1} & =2 \operatorname{tr} X \operatorname{tr} Y \\
\operatorname{tr} X Y^{2}+\operatorname{tr} X & =2 \operatorname{tr} X Y \operatorname{tr} Y .
\end{aligned}
$$

The general hypothesis in what follows is

$$
B, C \in \mathrm{SL}(2, \mathbb{R}), \quad B^{2}=C^{3}=-\mathbf{1}, \quad \text { tr } B C<-1 .
$$

Apart from the powers $B^{l}, C^{j}$, any element in the subgroup of $\operatorname{SL}(2, \mathbb{R})$ generated by $B, C$ is equivalent (i.e. conjugate or conjugate to the inverse) to a word $B C^{i_{1}} B C^{i_{2}} \cdots B C^{i_{n}}$ for some integer $n$ and exponents $i_{k} \in\{+1,-1\}$.

Proposition 7.1. For words $B C^{i_{1}} B C^{i_{2}} \cdots B C^{i_{n}}$ with $B, C$ as in (7.1) the following sign and monotonicity relations hold:

$$
\operatorname{sgn}\left(\operatorname{tr} B C^{i_{1}} B C^{i_{2}} \cdots B C^{i_{n}}\right)=\prod_{k=1}^{n}\left(-i_{k}\right) .
$$

For $n \geqslant 2$,

$$
\left|\operatorname{tr} B C^{i_{1}} B C^{i_{2}} \cdots B C^{i_{n-1}}\right|<\left|\operatorname{tr} B C^{i_{1}} B C^{i_{2}} \cdots B C^{i_{n}}\right| .
$$

Proof. We prove these assertions simultaneously by induction over $n$ using the above trace relations. For $n=1,2$, the traces are

$$
\begin{gathered}
\operatorname{tr} B C=-y, \quad \operatorname{tr} B C^{-1}=y, \\
\operatorname{tr} B C B C=\operatorname{tr} B C^{-1} B C^{-1}=2 y^{2}-1, \\
\operatorname{tr} B C B C^{-1}=\operatorname{tr} B C^{-1} B C=-\left(2 y^{2}-\frac{1}{2}\right) .
\end{gathered}
$$


Now let $n \geqslant 2$ and assume that (i) and (ii) hold for any word with a number of $B C^{i}$-pairs less than or equal $n$.

We first prove the sign rule for $B C^{i_{1}} \cdots B C^{i_{n}} B C^{i_{n+1}}$.

(1) Assume two (cyclically) adjacent exponents are equal, i.e. $i_{k}=i_{k+1}$ or $i_{n+1}=i_{1}$. As the sign rule is invariant under cyclic permutation, it suffices here to consider the case $i_{n}=i_{n+1}$. In this case,

$$
\begin{aligned}
\operatorname{tr} B C^{i_{1}} \cdots B C^{i_{n}} B C^{i_{n+1}} & =\operatorname{tr} B C^{i_{1}} \cdots B C^{i_{n-1}}\left(B C^{i_{n}}\right)^{2} \\
& =2 \operatorname{tr} B C^{i_{n}} \operatorname{tr} B C^{i_{1}} \cdots B C^{i_{n}}-\operatorname{tr} B C^{i_{1}} \cdots B C^{i_{n-1}} .
\end{aligned}
$$

By hypothesis, $\left|\operatorname{tr} B C^{i_{1}} \cdots B C^{i_{n-1}}\right|<\left|\operatorname{tr} B C^{i_{1}} \cdots B C^{i_{n}}\right|$. Since also $\left|\operatorname{tr} B C^{i_{n}}\right|>1$, the subtraction in the second line has no effect on the sign. Therefore

$$
\operatorname{sgn}\left(\operatorname{tr} B C^{i_{1}} \cdots B C^{i_{n}} B C^{i_{n+1}}\right)=\operatorname{sgn}\left(\operatorname{tr} B C^{i_{n}}\right) \operatorname{sgn}\left(\operatorname{tr} B C^{i_{1}} \cdots B C^{i_{n}}\right),
$$

and the sign rule follows.

(2) If we can't find two exponents as in (1), then the word is conjugate to $\left(B C B C^{-1}\right)^{k}$, where the sign rule is well known from Chebyshev polynomials or powers of hyperbolic elements.

For the proof of (ii) we consider three cases.

Case 1: $i_{n}=i_{n+1}$. Here the inequality follows from (*), $\left|\operatorname{tr} B C^{i_{n}}\right|>1$ and because by induction hypothesis, $\left|\operatorname{tr} B C^{i_{1}} \cdots B C^{i_{n-1}}\right|<\left|\operatorname{tr} B C^{i_{1}} \cdots B C^{i_{n}}\right|$.

Case 2: $i_{1}=i_{n+1}$. This is done using Case 1,

$$
\begin{aligned}
\left|\operatorname{tr} B C^{i_{1}} \cdots B C^{i_{n}}\right| & =\left|\operatorname{tr} B C^{i_{2}} \cdots B C^{i_{n}} B C^{i_{1}}\right|=\left|\operatorname{tr} B C^{i_{2}} \cdots B C^{i_{n}} B C^{i_{n+1}}\right| \\
& <\left|\operatorname{tr} B C^{i_{2}} \cdots B C^{i_{n+1}} B C^{i_{1}}\right|=\left|\operatorname{tr} B C^{i_{1}} \cdots B C^{i_{n}} B C^{i_{n+1}}\right| .
\end{aligned}
$$

Case 3: $i_{1}=i_{n}=-i_{n+1}$. Using the observation $C+C^{-1}=\mathbf{1}$ we compute

$$
\begin{aligned}
& B C^{i_{1}} \cdots B C^{i_{n-1}} B C^{i_{1}} B C^{-i_{1}} \\
& \quad+B C^{i_{1}} \cdots B C^{i_{n-1}} B C^{i_{1}} B C^{i_{1}}=B C^{i_{1}} \cdots B C^{i_{n-1}} B C^{i_{1}} B .
\end{aligned}
$$

Here the traces of the first and the second term have opposite signs, by (i). But the trace of the sum and the trace of the first term have the same signs:

$$
\begin{aligned}
\operatorname{sgn}\left(\operatorname{tr}\left(B C^{i_{1}} \cdots B C^{i_{n-1}} B C^{i_{1}} B\right)\right) & =\operatorname{sgn}\left(\operatorname{tr} B C^{i_{2}} \cdots B C^{i_{n-1}} B C^{-i_{1}}\right) \\
& =i_{1} \prod_{k=2}^{n-1}\left(-i_{k}\right) \\
& =\operatorname{sgn}\left(\operatorname{tr} B C^{i_{1}} \cdots B C^{i_{n-1}} B C^{i_{1}} B C^{-i_{1}}\right) .
\end{aligned}
$$

Hence,

$$
\left|\operatorname{tr} B C^{i_{1}} \cdots B C^{i_{n-1}} B C^{i_{1}} B C^{i_{1}}\right|<\left|\operatorname{tr} B C^{i_{1}} \cdots B C^{i_{n-1}} B C^{i_{1}} B C^{-i_{1}}\right| .
$$

By Case 1,

$$
\left|\operatorname{tr} B C^{i_{1}} \cdots B C^{i_{n-1}} B C^{i_{n}}\right|<\left|\operatorname{tr} B C^{i_{1}} \cdots B C^{i_{n-1}} B C^{i_{n}} B C^{i_{1}}\right|,
$$

and the inequality follows.

Returning to the missing point (iii) in the proof of Theorem 5.2, we note that Proposition 7.1 holds, of course, also for $H_{A D}$. Since $y$ is the absolute trace of $B C$, and $B C$ is in the same extended conjugacy class as $A D$, this shows that the first two lines in $\Lambda_{A D} \cup \Lambda_{B C}$ are $y$ and $2 y^{2}-1 / 2$. This completes the proof of Theorem 5.2. 
Let us also complete the proof of the main result, Theorem 1.3: By Corollary 4.12, the discrete groups with $\epsilon=-1$ are the elementary group of order 6 , whose spectrum is finite, and the $(2,3, n)$-triangle groups which are of the form $H_{B C}$. If $n$ is finite, then $|\operatorname{tr} B C|=$ $\cos (\pi / n)$ is the largest line $<1$ in the spectrum, and if $n=\infty$, then by Proposition $7 \cdot 1$, $|\operatorname{tr} B C|$ is the smallest line $\geqslant 1$. Hence these groups all have different spectra. Finally, zero, the trace of the order 2 element, occurs with multiplicity 1 in the spectrum of a triangle group but with multiplicity 2 in any 2233-Möbius group with $\epsilon=1$.

\section{Two examples}

For completeness we give here the parameters $(x, y, z) \in \mathcal{F}=\mathcal{F}_{1}$ for the two examples in [26]. We list, without giving proofs, the number field, the quaternion algebra and the two non-conjugate maximal orders that give rise to these examples. A more thorough description follows in the Appendix.

The examples are based on the totally real number field $k=\mathbb{Q}(\phi)$, where $\phi$ is a root of the polynomial $t^{3}-4 t+1$. The ring of integers in $k$ is

$$
R_{k}=\mathbb{Z}(\phi)=\mathbb{Z}+\mathbb{Z} \phi+\mathbb{Z} \phi^{2} .
$$

The quaternion algebra is $\mathcal{A}=H\left(\frac{a, b}{k}\right)$ with $a=-\phi, b=-2-\phi$. Thus $\mathcal{A}$ is the 4dimensional $k$-vector space

$$
\mathcal{A}=\mathbb{Q}(\phi) 1+\mathbb{Q}(\phi) I+\mathbb{Q}(\phi) J+\mathbb{Q}(\phi) K
$$

endowed with the associative multiplication induced by

$$
\begin{gathered}
I^{2}=a=-\phi, \quad J^{2}=b=-2-\phi, \quad K^{2}=-a b=-2 \phi-\phi^{2}, \\
I * J=-J * I=K .
\end{gathered}
$$

( 1 is central.) $\mathcal{A}$ is a division algebra and the smallest root $\phi=-2.1149 \ldots$ of $t^{3}-4 t+1$ induces an embedding of $\mathcal{A}$ into $\mathrm{M}(2, \mathbb{R})$ given by

$$
I \longmapsto \sqrt{a}\left(\begin{array}{rr}
1 & 0 \\
0 & -1
\end{array}\right), \quad J \longmapsto \sqrt{b}\left(\begin{array}{ll}
0 & 1 \\
1 & 0
\end{array}\right), \quad K \longmapsto \sqrt{a b}\left(\begin{array}{rr}
0 & 1 \\
-1 & 0
\end{array}\right) .
$$

Two non-conjugate maximal orders in $\mathcal{A}$ can be given as follows: The first order, $\mathcal{O}_{1}$, is the $\mathbb{Z}(\phi)$-module generated by $1, A, B, \eta$, where

$$
A=-\frac{1}{2} J+\frac{1}{2}(2-\phi) K, \quad B=\frac{1}{2} J+\frac{1}{2}(2-\phi) K, \quad \eta=B * A .
$$

The corresponding group $\mathcal{O}_{1}^{1}$ of elements of norm 1 in $\mathcal{O}_{1}$ is generated by $A, B, C, D$, where $A$ and $B$ are as just defined and

$$
C=(2-\phi) \phi(1+\eta)-(\phi-1) B, \quad D=C^{-1} * B * A .
$$

(In the embedding (8.1) the resulting generators of the Fuchsian group do not have the normalized position as in Section 3.)

The second order, $\mathcal{O}_{2}$, is the $\mathbb{Z}(\phi)$-module generated by $K, P, Q, \eta^{\prime}$, where

$$
P=1+J, \quad Q=\frac{1}{2} 1+\frac{1}{2} I+\frac{1}{2}(\phi+1)(\phi-2) K, \quad \eta^{\prime}=\frac{1}{2}(\phi-1) 1+\frac{1}{2} \phi J+\frac{1}{2} K .
$$

As an alternative we may also take the $\mathbb{Z}(\phi)$-module generated by $1, P, Q, \eta^{\prime}$ with

$$
P=\frac{1}{2} 1+\frac{1}{2} I+\left(\phi^{2}-\phi\right) \frac{1}{2} K, \quad Q=\frac{1}{2}\left(\phi^{2}-1\right) 1+\frac{1}{2} J-\phi^{2} \frac{1}{2} K, \quad \eta^{\prime}=(\phi-2) K .
$$


Either way, the corresponding group $\mathcal{O}_{2}^{1}$ of elements of norm 1 in $\mathcal{O}_{2}$ is generated by $A^{\prime}, B^{\prime}, C^{\prime}, D^{\prime}$, where

$$
\begin{aligned}
& A^{\prime}=\frac{1}{2}\left(4-\phi-\phi^{2}\right) I+\frac{1}{2}\left(-2 \phi+\phi^{2}\right)(J+K) \\
& B^{\prime}=\frac{1}{2}\left(-1-\phi+\phi^{2}\right)(J+K) \\
& C^{\prime}=\frac{1}{2}+\frac{1}{2} I+\left(-2 \phi+\phi^{2}\right) J+\frac{1}{2}\left(2-3 \phi+\phi^{2}\right) K \\
& D^{\prime}=C^{\prime-1} * B^{\prime} * A^{\prime} .
\end{aligned}
$$

The parameters of the corresponding arithmetic Fuchsian 2233-groups $\Gamma_{1}, \Gamma_{2}$, when $\phi$ is interpreted as the smallest root of $t^{3}-4 t+1$, are as follows.

For $\Gamma_{1}$ :

$$
\begin{aligned}
x & =-\operatorname{tr} \eta=\frac{1}{4} b-\frac{1}{4}(2-\phi)^{2}(-a b)=\frac{1}{4}\left(-2-\phi+(2-\phi)^{2} \phi(\phi+2)\right)=-\frac{1}{2} \phi \\
& =1.0574 \ldots \\
y & =-\phi+1=3.1149 \ldots \quad z=x y=\frac{1}{2}\left(\phi^{2}-\phi\right)=3.2938 \ldots
\end{aligned}
$$

For $\Gamma_{2}$ :

$$
x=y=z=-\frac{1}{2} \phi+\frac{1}{2}=1.5574 \ldots
$$

The second example is depicted as point $m_{2}$ on the boundary of the domain in Figure 2. Point $m_{1}$ corresponding to the first example lies on the first boundary arc but is outside the scope of the figure. By Theorem 5.2, the smallest value of $\operatorname{tr}=(1 / 2) \mid$ trace $\mid$ for the hyperbolic elements in $\Gamma_{1}$ is $-(1 / 2) \phi$, while for $\Gamma_{2}$ this value is $-(1 / 2) \phi+1 / 2$.

That $-(1 / 2) \phi$ occurs in one of the spectra but not in the other can also be seen by looking at the quadratic field extension $k(u)$, where $u$ is a root of the polynomial $t^{2}-\phi t+1$. This is done in a more general analysis in the Appendix.

\section{Appendix}

The main result in [26] states:

THEOREM 9-1. There exists a pair of isospectral non-isometric hyperbolic 2-orbifolds $\mathbb{H}^{2} / \Gamma_{1}, \mathbb{H}^{2} / \Gamma_{2}$ where $\Gamma_{1}, \Gamma_{2}$ have signature $(0 ; 2,2,3,3 ; 0)$.

In view of Theorem 1.3, this theorem cannot be correct. The method in the proof in [26] was to use arithmetic Fuchsian groups. More precisely, it was a construction of a quaternion algebra $A$ which contained two non-conjugate maximal orders $\mathcal{O}_{1}, \mathcal{O}_{2}$ giving rise to non-conjugate Fuchsian groups $P \rho\left(\mathcal{O}_{1}^{1}\right), P \rho\left(\mathcal{O}_{2}^{1}\right)$. We then claimed that these groups were isospectral, basing our claim on methods similar to those applied in $[35,36]$ where the following result is proved:

THEOREM 9.2. There exist pairs of isospectral non-isometric hyperbolic compact 2manifolds.

Unfortunately, our claim is false as the pair $P \rho\left(\mathcal{O}_{1}^{1}\right), P \rho\left(\mathcal{O}_{2}^{1}\right)$ can be shown to be nonisospectral. This was drawn to our attention by the first authors of this paper who directly constructed these groups and used their geometric methods to show that they were not isospectral. The error in our method is due to the existence of a subtle condition called selectivity concerning embeddings of commutative orders in maximal orders in quaternion algebras and recently enunciated in [9]. Using this condition, it can be shown that our non-conjugate 
groups $P \rho\left(\mathcal{O}_{1}^{1}\right), P \rho\left(\mathcal{O}_{2}^{1}\right)$ are forced to be non-isospectral. It is the purpose of this appendix to clarify this aspect in a wider context than these two groups alone.

Recall that arithmetic Fuchsian groups arise as follows: let $k$ be a totally real number field and $A$ a quaternion algebra over $k$ which is ramified at all real places except one. Thus there is a representation $\rho: A \rightarrow \mathrm{M}(2, \mathbb{R})$. If $\mathcal{O}$ is an order in $A$ and $\mathcal{O}^{1}$ denotes the elements of norm 1 in $\mathcal{O}$, then $P \rho\left(\mathcal{O}^{1}\right)$ is a Fuchsian group which, furthermore, will be cocompact if $A$ is a division algebra. The set of all Fuchsian groups commensurable with some such $P \rho\left(\mathcal{O}^{1}\right)$ is the set of all arithmetic Fuchsian groups (see e.g. [36]). We recall that the isomorphism class of a quaternion algebra $A$ over $k$ is determined by its ramification set $\operatorname{Ram}(A)$. This is a finite subset of even cardinality of the set $\Lambda$ of all places $v$ of $k$ defined by

$$
\operatorname{Ram}(A)=\left\{v \in \Lambda \mid A \otimes_{k} k_{v} \text { is a division algebra }\right\}
$$

where $k_{v}$ is the completion of $k$ at the valuation given by $v$. As noted above, for arithmetic Fuchsian groups, all archimedean places of $k$ are real and all but one of these belong to $\operatorname{Ram}(A) . \operatorname{Ram}(A)$ may also, of course, contain $\mathcal{P}$-adic places. We let $\operatorname{Ram}_{\infty}(A), \operatorname{Ram}_{f}(A)$ denote the subsets of real and finite $(\mathcal{P}$-adic) ramified places respectively.

We will assume throughout that $A$ and $k$ will be such that they define cocompact arithmetic Fuchsian groups although most results given below hold in a wider context. Thus $k$ will be totally real, $A$ will be ramified at all real places except one and $A$ is a division algebra. Suppose that $P \rho\left(\mathcal{O}^{1}\right)$ has an element $\gamma_{0}=P \rho\left(x_{0}\right)$ and trace $\left(x_{0}\right)=t_{0}$. Then $t_{0} \in R_{k}$, the ring of integers in $k$. Then if we define the quadratic extension $L=k\left(u_{0}\right)$ of $k$ where $u_{0}$ satisfies $x^{2}-t_{0} x+1=0$, there is an embedding $\sigma: L \rightarrow A$ induced by $\sigma\left(u_{0}\right)=x_{0}$. In general, there are well-established necessary and sufficient conditions for a quadratic extension to embed in a quaternion algebra (see e.g. [36, theorem 3.8]):

THEOREM 9.3. Let $A$ be a quaternion algebra over the number field $k$ and let $L$ be a quadratic extension of $k$. Then $L$ embeds in $A$ if and only if $L \otimes_{k} k_{v}$ is a field for each $v \in \operatorname{Ram}(A)$.

Let $\Omega$ denote the commutative $R_{k}$-order $R_{k}+R_{k} u_{0}$ so that $\Omega \subset L$. With $L \subset A, A=$ $L a+L b$ for some $a, b \in A$. Then $R_{L} a+R_{L} b=I$ is an ideal in $A$. If $\mathcal{O}_{\ell}(I)$ denotes the order on the left of $I$, i.e.

$$
\mathcal{O}_{\ell}(I)=\{\alpha \in A \mid \alpha(I) \subset I\},
$$

then $\Omega \subset R_{L} \subset \mathcal{O}_{\ell}(I) \subset \mathcal{O}$ for some maximal order $\mathcal{O}$. Thus the embedding $\sigma: L \rightarrow$ $A$ described above, yields $\sigma(\Omega) \subset \mathcal{O}$. Conversely, any embedding $\sigma: L \rightarrow A$ such that $\sigma(\Omega) \subset \mathcal{O}$, yields an element in $\mathcal{O}^{1}$ of trace $t_{0}$. If we denote this set of embeddings by $\mathcal{E}_{\mathcal{O}}(L)$, then the number of conjugacy classes of elements in $\mathcal{O}^{1}$ of trace $t_{0}$ is the cardinality of the set $\mathcal{E}_{\mathcal{O}}(L) / \mathcal{O}^{1}$ where $\mathcal{O}^{1}$ acts by conjugation. When $\gamma_{0}$ is hyperbolic, this cardinality is the multiplicity of the number $\ell\left(\gamma_{0}\right)$ in the spectrum of $P \rho\left(\mathcal{O}^{1}\right)$.

The general problem of embedding commutative orders in maximal orders in a quaternion algebra over a number field was solved in [9]. Restricted to the class of quaternion algebras being considered here, the following theorem holds:

THEOREM 9.4. Let A be a quaternion algebra over the number field $k$. Let $\Omega$ be a commutative $R_{k}$-order contained in $A$ whose field of quotients $L$ is a quadratic extension of $k$. Then every maximal order $\mathcal{O}$ of A contains a conjugate of $\Omega$ except when the following conditions both hold: 
(a) the extension $L \mid k$ and the algebra $A$ are unramified at all finite places and ramified at exactly the same set of real places;

(b) all prime ideals dividing the relative discriminant ideal $d_{\Omega \mid R_{k}}$ of $\Omega$ are split in $L \mid k$.

Now suppose that (a) and (b) hold. Then A has an even number of conjugacy classes of maximal orders and the maximal orders containing some conjugate of $\Omega$ make up exactly half of these classes.

Definition 9.5. If conditions (a) and (b) hold, then $\Omega$ is said to be selective for $A$.

Thus when $\Omega$ corresponds to a number in the spectrum, that number will appear in the spectrum of each $P \rho\left(\mathcal{O}^{1}\right)$ for $\mathcal{O}$ any maximal order, provided $\Omega$ is not selective for $A$. This selective condition was not enunciated in $[35,36]$, and so certain results in [36], to quote [9], "must be corrected to account for selective orders". There are formulae in [36, section 5.5] for counting the number of embeddings. Using this, or by a more direct method (see [25, theorem $12 \cdot 4 \cdot 5]$ ), we obtain:

THEOREM 9.6. Let $A$ and $\Omega$ be as described in Theorem 9.4. Suppose that condition (a) of selectivity fails to hold. Then the cardinality of the set $\mathcal{E}_{\mathcal{O}}(L) / \mathcal{O}^{1}$ is independent of the choice of maximal order.

THEOREM 9.7. Let $A$ be a quaternion algebra over the field $k$. Let $\mathcal{O}_{1}, \mathcal{O}_{2}$ be maximal orders in $A$. If $A$ has finite ramification, then the orbifolds $\mathbb{H}^{2} / P \rho\left(\mathcal{O}_{1}^{1}\right), \mathbb{H}^{2} / P \rho\left(\mathcal{O}_{2}^{1}\right)$ are isospectral.

Proof. This follows immediately from Theorems 9.4 and 9.6 as condition (a) of selectivity fails under the assumption on $A$.

THEOREM 9.8. Let $A$ be a quaternion algebra over the field $k$. Let $\mathcal{O}$ be a maximal order in A. If $P \rho\left(\mathcal{O}^{1}\right)$ does not contain elements of both orders 2 and 3 , then $A$ has finite ramification.

Proof. Suppose that $A$ has no finite ramification. Let $\xi$ denote a primitive 4 th or 6 th root of unity. Since $k$ is totally real, $k(\xi) \otimes_{k} k_{v}$ is a field for each $v \in \operatorname{Ram}_{\infty}(A)=\operatorname{Ram}(A)$. Thus, by Theorem $9 \cdot 3, k(\xi)$ embeds in $A$ and $k(\xi) \mid k$ is ramified at every real place. Thus condition (a) of selectivity fails, so that $\Omega=R_{k}(\xi)$ embeds in every maximal order. Thus $P \rho\left(\mathcal{O}^{1}\right)$ contains elements of order 2 and of order 3 .

COROLLARY 9.9. Let $A$ be a quaternion algebra over the field $k$ and let $\mathcal{O}_{1}, \mathcal{O}_{2}$ be maximal orders in A. If $P \rho\left(\mathcal{O}_{1}^{1}\right)$ is torsion free, then the hyperbolic 2-manifolds $\mathbb{H}^{2} / P \rho\left(\mathcal{O}_{1}^{1}\right)$, $\mathbb{H}^{2} / P \rho\left(\mathrm{O}_{2}^{1}\right)$ are isospectral.

Proof. Note that, by the argument in the preceding theorem, if $P \rho\left(\mathcal{O}_{1}^{1}\right)$ is torsion free, so is $P \rho\left(\mathcal{O}_{2}^{1}\right)$. The result then follows from Theorems 9.7 and $9 \cdot 8$.

To obtain Theorem 9.2 above, quaternion algebras were constructed in [35] such that the groups $P \rho\left(\mathcal{O}_{1}^{1}\right)$ were torsion free and the type number, i.e. the number of conjugacy classes in $A^{*}$ of maximal orders in $A$, was greater than one. This then forces the groups $P \rho\left(\mathcal{O}_{1}^{1}\right)$, $P \rho\left(\mathcal{O}_{2}^{1}\right)$, for $\mathcal{O}_{1}, \mathcal{O}_{2}$ from different conjugacy classes, to be non-conjugate. This method can then be applied more generally to hyperbolic 2-orbifolds, provided the groups $P \rho\left(\mathcal{O}^{1}\right)$ do not contain elements of orders 2 and 3. 
Now consider the example used in [26]. Let $k=\mathbb{Q}(\phi)$ where $\phi=x_{1}$ is a root of the polynomial $x^{3}-4 x+1$ which has 3 real roots $x_{1}, x_{2}, x_{3}$ where

$$
x_{1}<-2<0<x_{2}<1<x_{3}<2 .
$$

Note that $\phi, \phi+2, \phi-2$ are all units and we can take $\phi, \phi+2$ as a fundamental system. Let $A$ be the quaternion algebra over $k$ ramified only at the two real places $v_{2}, v_{3}$ corresponding to the roots $x_{2}, x_{3}$. The type number of $A$ works out to be 2 .

Let $L=k(u)$ where $u$ satisfies $x^{2}-\phi x+1=0$. The discriminant of this polynomial is $\phi^{2}-4$, which is negative at $v_{2}, v_{3}$. Thus, by Theorem $9 \cdot 3, L$ embeds in $A$ and so $\Omega=R_{k}[u]$ embeds in $A$. Note that, if $y$ is the image of $u$, then $\gamma=P \rho(y)$ is a hyperbolic element, where $\rho$ is a $k$-embedding of $A$ in $\mathrm{M}(2, \mathbb{R})$. Now $\Omega$ is selective for $A$. This follows since $\phi^{2}-4$ is a unit in $R_{k}$ so that $L \mid k$ is unramified at all finite places as well as being ramified at exactly the real places $v_{2}, v_{3}$. Since $\Omega=R_{L}$, no prime ideals divide the relative discriminant ideal. Since the type number of $A$ is 2 , we choose $\mathcal{O}_{1}, \mathcal{O}_{2}$ to be non-conjugate maximal orders. Now $\Omega$ embeds in exactly one of these, thus showing that $P \rho\left(\mathcal{O}_{1}^{1}\right), P \rho\left(\mathcal{O}_{2}^{1}\right)$ are not isospectral. In conclusion, note that these groups necessarily have elements of orders 2 and 3 and do not have any elements of higher order since $2 \cos \pi / n \notin k$ for $n \geqslant 4$. In addition, their covolume is given by the formula

$$
\frac{8 \pi \Delta_{k}^{3 / 2} \zeta_{k}(2) \prod_{\mathcal{P} \in \operatorname{Ram}_{f}(A)}(N \mathcal{P}-1)}{\left(4 \pi^{2}\right)^{3}}
$$

which can be computed as approximately $2 \pi(0.3333)$ (e.g. using [10]). Thus their signature must be $(0 ; 2,2,3,3 ; 0)$.

Acknowledgements. The authors were supported by the Swiss National Science Foundation, FNS-grants 20-68181, 20-101639, 21-65270.

\section{REFERENCES}

[1] A. F. BEARDon. The geometry of discrete groups. Graduate Texts in Mathematics, vol. 91. (SpringerVerlag, 1995).

[2] A. BinotTo. Surfaces de Riemann de type $(0,3)$ et $(1,1)$ : Polynômes au service de l'étude spectrale. Ph.D.thesis, École Polytechnique Fédérale de Lausanne (2000).

[3] R. Brooks. Constructing isospectral manifolds. Amer. Math. Monthly 95 (1988), 823-839.

[4] R. Brooks, R. Gornet and W. H. Gustafson. Mutually isospectral Riemann surfaces. Adv. Math. 138 (1998), 306-322.

[5] R. BRoOKS and R. TSE. Isospectral surfaces of small genus. Nagoya Math. J. 107 (1987), 13-24.

[6] P. BUSER. Isospectral Riemann surfaces. Ann. Inst. Fourier (Grenoble) 36 (1986), 167-192.

[7] P. BUSER. Geometry and spectra of compact Riemann surfaces. Progr. Math. 106 ( Birkhäuser, 1992).

[8] P. Buser and K.-D. SEMmLER. The geometry and spectrum of the one holed torus. Comment. Math. Helv. 63 (1988), 259-274.

[9] T. Chinburg and E. Friedman. An embedding theorem for quaternion algebras. J. London Math. Soc. 60 (1999), 33-44.

[10] H. Cohen. PARI-GP: A software package for computer-aided number theory. Available at http://www.parigp-home.de/ (2001)

[11] G. DE RHAM. Sur les polygones générateurs de groupes fuchsiens. Enseignement Math. 17 (1971), 49-61.

[12] R. Dianu. Sur le spectre des tores pointés. Ph.D.thesis, École Polytechnique Fédérale de Lausanne (2000).

[13] B. Fine and G. Rosenberger. Classification of all generating pairs of two generator Fuchsian groups. Groups '93, Galway/St Andrews. London Math. Soc. Lecture Notes, vol. 211 (1995), 205232.

[14] B. Fine, G. Rosenberger and M. Stille. Nielsen transformations and applications: a survey. Groups-Korea '94 (Pusan), (de Gruyter, 1995), 69-105. 
[15] R. FRICKE and F. KLEIN. Vorlesungen über die Theorie der Automorphen Funktionen. Vol. 1 (Teubner, 1897).

[16] D. GRIFFITHS. Length inequalities for systems of geodesic loops on a surface of genus two. Bull. London Math. Soc. 28 (1996), 505-508.

[17] D. GRIFFITHS. The side-pairing elements of Maskit's fundamental domain for the modular group in genus two. Ann. Acad. Sci. Fenn. Math. 26 (2001), 3-50.

[18] A. HAAS. Length spectra as moduli for hyperbolic surfaces. Duke Math. J. 52 (1985), 923-934.

[19] D. Hejhal. The Selberg trace formula for PSL $(2, \mathbb{R})$, vol. 1. Lecture Notes in Math., vol. 548, (Springer-Verlag, 1976).

[20] H. HeLLING. Diskrete Untergruppen von $\mathrm{SL}_{2}(\mathbb{R})$. Invent. Math. 17 (1972), 217-229.

[21] H. Helling. Über den Raum der kompakten Riemannschen Flächen vom Geschlecht 2. J. Reine Angew. Math. 268/269 (1974), 286-293.

[22] R. D. Horowitz. Characters of free groups represented in the two-dimensional special linear group. Comm. Pure Appl. Math. 25 (1972), 635-649.

[23] R. N. Kahlia and G. Rosenberger. Automorphisms of the Fuchsian groups of type $(0 ; 2,2,2, \mathrm{q} ; 0)$. Comm. Algebra 11 (1978), 1115-1129.

[24] J. LEHNER. On polygon groups, in: Analytic Number Theory (Philadelphia, 1980). Lecture Notes in Math., vol. 899, pp. 315-324 (Springer-Verlag, 1981).

[25] C. MACLACHLAN and A. REID. The arithmetic of hyperbolic 3-manifolds. Graduate Texts in Mathematics (Springer-Verlag, New York, 2003).

[26] C. Maclachlan and G. Rosenberger. Small volume isospectral, non-isometric, hyperbolic 2orbifolds. Arch. Math. 62 (1994), 33-37.

[27] B. MASKIT. Kleinian groups. Grundlehren der Mathematischen Wissenschaften, vol 287 (SpringerVerlag, 1988).

[28] B. MASKIT. A picture of moduli space. Invent. Math. 126 (1996), 341-390.

[29] J. MiLnOR. On the 3-dimensional Brieskorn manifolds M(p,q,r). Ann. Math. Studies 84 (1975), 175225.

[30] K.-D. SemmLER. An explicit fundamental domain for the Teichmüller space of Riemann surfaces of genus 2. Ph.D.thesis, École Polytechnique Fédérale de Lausanne (2000).

[31] M. SEPPÄLÄ and T. SORVALI. Geometry of Riemann surfaces and Teichmüller spaces, vol. 169 North-Holland Mathematics Studies (North-Holland Publishing Co., 1992).

[32] D. Singerman. Finitely maximal Fuchsian groups. J. London Math. Soc. 6 (1972), 29-38.

[33] T. SunADA. Riemannian coverings and isospectral manifolds. Ann. of Math. 121 (1985), 169-186.

[34] R. M. TSE. A lower bound for the number of isospectral surfaces; in: Recent developments in geometry (Los Angeles, 1987), pp. 161-164 (Amer. Math. Soc., 1989).

[35] M.-F. VignéRAS. Variétés riemanniennes isospectrales et non isométriques. Ann. of Math. 112 (1980), 21-32.

[36] M.-F. VignéRAs. Arithmétique des algèbres de quaternions. Lecture Notes in Math., vol. 800 (Springer-Verlag, 1980). 